\title{
A new species of Lipotrapeza (Echinodermata: Holothuroidea: Dendrochirotida: Phyllophoridae) from Japan, with a partial redescription of the syntypes of L. japonica
}

\author{
YUSUKE YAMANA $^{1, *} \&$ HisANORI KOHTSUKA ${ }^{2}$ \\ ${ }^{1}$ Wakayama Prefectural Museum of Natural History, Funo 370-1, Kainan, Wakayama 642-0001, Japan \\ ${ }^{2}$ Misaki Marine Biological Station, School of Science, University of Tokyo, 1024 Koajiro, Misaki, Miura, Kanagawa 238-0225, \\ Japan
}

Received 1 March 2017; Accepted 31 May 2017 Responsible Editor: Shigeaki Kojima

\begin{abstract}
A new dendrochirotid sea cucumber collected in the intertidal zone of western Japan, Lipotrapeza littoralis sp. nov., is described. This species has a medium body size (approximately $30-80 \mathrm{~mm}$ ), brownish body color, 20 tentacles arranged in a double circle $(15+5)$, a long calcareous ring, ten anal papillae and five anal scales, and numerous pedicels covering the entire body at an even density. For comparison, we also examined the syntype specimens of Lipotrapeza japonica Heding and Panning, 1954 and provide illustrations of the general appearance of its body, calcareous ring, and ossicles. The calcareous rings of both species are very similar in appearance, but the new species is readily distinguishable from L. japonica by the number of anal papillae. The appearance of the rosette ossicles also differs in the two species.
\end{abstract}

Key words: Holothuroidea, Japan, Lipotrapeza japonica Heding and Panning, 1954, Lipotrapeza littoralis sp. nov., Phyllophoridae

\section{Introduction}

The dendrochirotid sea-cucumber genus Lipotrapeza Clark, 1938 [type species: Phyllophorus vestiens Joshua, 1914] is one of a small group of genera included in the Phyllophoridae, and it is presently comprised of six species (Paulay 2015). Clark's (1938) diagnosis was as follows: 20 expansible tentacles (10 large and 10 small), a stout calcareous ring (with paired short tapered posterior composite prolongations), body wall lacking ossicles in the middle part (numerous rosette ossicles and perforated rod ossicles in the introvert and also in some other parts), pedicels scattered without definite arrangement (with end plate ossicles and perforated ossicles), and table ossicles occurring at the anal tip in some individuals. Clark (1938) considered the calcareous ring the most important diagnostic character of this genus. Additional diagnostic information concerning the ossicles was provided by O'Loughlin et al. (2012): body wall of young individuals containing ossicles (table

*Corresponding author: Yusuke Yamana; E-mail, yamanamako@gmail. com ossicles with spires consisting of four pillars).

In Japanese waters, only one nominal species of Lipotrapeza has been reported: Lipotrapeza japonica Heding and Panning, 1954, found at a depth of 60 fathoms in $34^{\circ} 20^{\prime} \mathrm{N}, 130^{\circ} 10^{\prime} \mathrm{E}$ (i.e., at $c a .110 \mathrm{~m}$ in the Genkainada Sea, western Japan). However, O'Loughlin et al. (2012) questioned the inclusion of this species in the genus Lipotrapeza because Heding and Panning (1954) reported that L. japonica had 20 tentacles in triple circles (five large pairs, five small, and five small) and a calcareous ring with long discrete paired posterior prolongations. These features have not been observed in other species of Lipotrapeza. Unfortunately, Heding and Panning (1954) provided only a few sketches of the ossicles and the calcareous ring shape, and no images or sketches of the body shape, and neither researcher observed L. japonica again. Clear images to support the original description of this species have therefore not been available to date, and detailed information on the tentacles, ossicles, calcareous ring, and body shape are needed to determine the precise placement of this species.

Recently, we found a phyllophorid species from in the 
intertidal zone in western Japan. This species mostly agreed with Clark's (1938) diagnosis of Lipotrapeza and was different not only from L. japonica but also from all its congeners. Herein, we describe this species as new to science. For comparison, we examined the syntypes of $L$. japonica in the Zoological Museum, University of Copenhagen (ZMUC), Denmark, partial redescriptions of which are also given.

\section{Materials and Methods}

All the specimens were collected by hand from sand and gravel sediment under boulders in the lower intertidal zone at the following sites in western Japan: (BOJ) Bojima Island, Kujukushima Islands, near the west coast of Nagasaki Prefecture (33 $15^{\prime} 13^{\prime \prime} \mathrm{N}, 129^{\circ} 33^{\prime} 25^{\prime \prime} \mathrm{E}$ ), March 28, 2013 (coll. Y. Yamana); (ATA) Atata coast, Hirao Bay, east coast of Yamaguchi Prefecture $\left(33^{\circ} 54^{\prime} 35.0^{\prime \prime} \mathrm{N}, 132^{\circ} 02^{\prime} 40.5^{\prime \prime} \mathrm{E}\right)$, January 2, 2014 (coll. Y. Yamana); (TOS) Tosaka coast, Wakaura Bay, north coast of Wakayama Prefecture $\left(34^{\circ} 08^{\prime} 18.4^{\prime \prime} \mathrm{N}\right.$, $135^{\circ} 08^{\prime} 09.9^{\prime \prime} \mathrm{E}$ ), July 25, 2014 (coll. Y. Yamana).

We fixed and preserved the specimens in $80 \%$ ethanol. Some specimens were fixed after anesthetization in a menthol solution $(0.37 \mathrm{~g}$ of menthol dissolved into $100 \mathrm{~mL}$ of $99 \%$ ethanol, this then diluted to $3.0 \%$ with seawater) for 30 minutes. When the anesthesia had taken effect, the tentacles emerged in response to a gentle squeeze of the body. Before fixation, hydrated magnesium chloride $\left(\mathrm{MgCl}_{2} \cdot 6 \mathrm{H}_{2} \mathrm{O}\right)$ was added to the solution at a concentration of $3.0 \mathrm{~g}$ per $100 \mathrm{~mL}$ for further anesthetization.

All the type specimens were dissected, and the tentacles, anal appendages, Polian vesicles, stone canals, and calcareous rings were examined under a stereoscopic dissecting microscope (Nikon SMZ). In order to observe the morphology of the ossicles, small pieces of tissue were removed from the following parts of the body: 1) the midparts of the inter-radial tentacle on the abdominal-lateral side; 2) the peri-oral skin on the abdominal-lateral side; 3) the skin of the esophagus on the abdominal-lateral side; 4) the middle part of the skin of the introvert in the interradius, on the abdominal-lateral side; 5) the anterior, 6) middle, and 7) posterior parts of the body-wall integument in the inter-radius on the abdominal side; $8-10$ ) the integument of the same three regions on the dorsal side; 11-13) the pedicels of the same three regions of the abdominal side in the radius; and 14) appendage and skin around the anus on the abdominal-lateral side.

The tissue samples were dissolved in sodium hypochlorite $(\mathrm{NaClO}, 5 \%)$ under a compound microscope (Nikon Optiphot). Ossicles of all the type specimens were counted and measured using photographs. We classified the ossicles into six types: two types of "rosettes" [i.e., rosette-like ossicles, extremely branched and perforated: 1) rosettes and 2) small rosettes], one type of "table" [ossicles consisting of a multi-perforate disc and a skeleton-forming spire that grows perpendicularly from the disc: 3) tables], one type of "rod" [rod-like ossicles armed distally with lumps or branches or a few perforations: 4) rods], and two types of "plates" [multi-perforate oval plate ossicles having curved surfaces: 5) supporting plates; and multi-perforate circular plate ossicles situated on the tips of pedicels: 6) endplates]. For every tissue sample, we measured the ossicles to the nearest micrometer using photographs.

The type specimens of the new species and glass slides of their ossicles have been deposited in the Invertebrate Collection (INV) of the Wakayama Prefectural Museum of Natural History (WMNH), in Kainan, Wakayama, Japan.

For comparison, we examined the syntypes of Lipotrapeza japonica in the Zoological Museum, University of Copenhagen (ZMUC), Denmark. One of the syntype specimens (the large specimen) had already been dissected by Heding and Panning (1954); we were therefore able to examine the Polian vesicles, the stone canals, and the calcareous rings without additional dissection, but to observe the tentacles, we had to dissect the oral area. To observe the ossicles of the syntypes of $L$. japonica, small pieces of tissue were taken from a tentacle, the peri-oral skin including the esophageal skin (we failed to separate the esophageal skin, and it was contaminated with peri-oral skin), the introvert, and the body wall near the middle inter-radius of the abdominal side; a pedicel from the middle abdominal side was also taken. In the large specimen, however, the whole of the anal periphery has already been removed by Heding and Panning (1954); we therefore took small pieces of tissue from the anal periphery, including an anal papilla, a pedicel, and a minute piece of mid-abdominal skin, from the other specimen, which had not been dissected.

To compare the two species, we tried to evaluate the appearance of the rosette ossicles, by comparing the total number of free-ends of branches in a rosette $(N f)$ with the approximate size of the rosette, based on sampling 10 typical rosettes at random from each tissue. As an approximation for the area $(A)$ of a rosette, which is roughly an oval with long axis $L$ and short axis $B$, we calculated $A=\pi L B / 4$, where $L$ and $B$ denote the length and width $(\mu \mathrm{m})$ of the same rosette, respectively. In the present study, the freeend of a branch was defined as: a free tip with its length more than half of its width.

\section{Taxonomy}

Order Dendrochirotia Grube, 1840

Family Phyllophoridae Östergren, 1907

Genus Lipotrapeza Clark, 1938

Lipotrapeza japonica Heding and Panning, 1954

(Figs. 1A, B; 2A, B)

Lipotrapeza japonica Heding and Panning, 1954: 176-178,

Abb. 85; Imaoka 1995: 554.

Material examined. Two syntypes: ZMUC-SYN-101, 18 May 1914, 60 Fathoms in $34^{\circ} 20^{\prime} \mathrm{N}, 130^{\circ} 10^{\prime} \mathrm{E}$ (i.e., at $c a$. $110 \mathrm{~m}$ in the Genkainada Sea, western Japan), collected by 
Dr Th. Mortensen. Large specimen (length $23 \mathrm{~mm}$, width $7 \mathrm{~mm}$ ) (Fig. 1A) with original dissection by Heding and Panning (1954) on its mid-abdominal to right side (approxi-

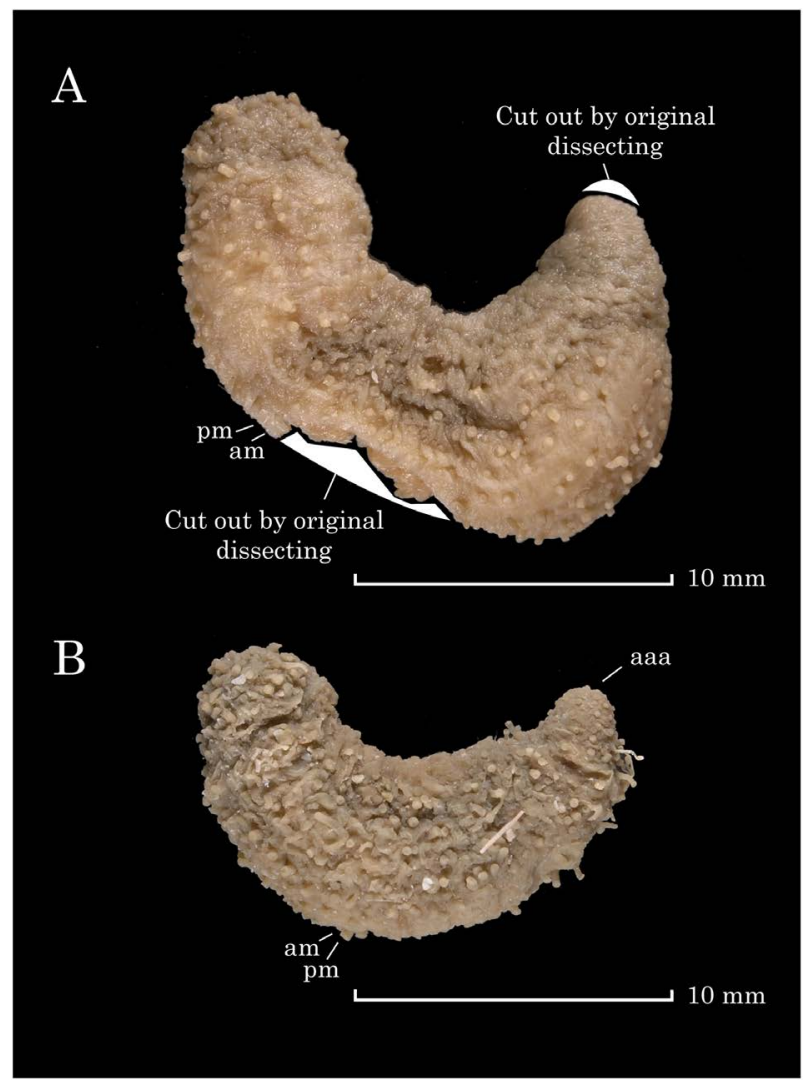

Fig. 1. Lateral views, Lipotrapeza japonica Heding and Panning, 1954. A, B, ZMUC-SYN-101 large and small specimens (syntypes). Abbreviations indicate sites of tissue sampling for ossicle examination: aaa, anal appendage and anal integument on abdominal-lateral side; am, middle part of body on abdominal side; pm, pedicels from middle part of body. mately $20-30 \%$ of skin removed) and anal periphery (whole anal periphery removed). Small specimen (length $17 \mathrm{~mm}$, width $5 \mathrm{~mm}$ ) (Fig. 1B) without any dissection.

Description. Body curved or U-shaped, with both ends turned upwards; body wall soft and thick. Body (preserved) solid yellowish-brown in color (Fig. 1). Retractable pedicels covering entire body at an even density. Color of pedicels (preserved) same as adjacent body. Pedicels becoming gradually smaller anterior and posterior to the middle portion of the body, absent in introvert region.

Twenty tentacles arranged in double circle $(15+5)$, including five pairs of large inter-radial tentacles and five single small radial tentacles in the outer circle and five very small radial tentacles in the inner circle. Short processes present around entrance of esophagus. Color of tentacles and introvert (preserved) solid whitish-yellow and paler yellowish-brown, respectively. Five anal papillae and five anal scales in radii (five anal papillae in outer circle and five anal scales in inner circle). Each anal scale containing a long calcareous structure.

Long calcareous ring present, encircling esophagus (Fig. 2A). Pieces of radials and inter-radials elements not forming a mosaic pattern. Radial main plates rectangular, notched anteriorly and posteriorly; inter-radial main plates sagittate, sharply protruding anteriorly. Radials with paired posterior prolongations, long. One Polian vesicle and one stone canal (Table 1). Gonad bundles composed of short and branched tubules.

Two types of ossicles present in tentacle of large specimen, viz., "rosettes" 33-119 $\mu \mathrm{m}$ long and $13-84 \mu \mathrm{m}$ wide, and "rods" $33-156 \mu \mathrm{m}$ long and $2-13 \mu \mathrm{m}$ wide at center of stem (Fig. 2A, Table 2). A few rosettes enlarged and swollen. Single type of ossicle present in peri-oral skin and esophageal processes of large specimen, viz., "rosettes" 11$51 \mu \mathrm{m}$ long and 8-28 $\mu \mathrm{m}$ wide (Fig. 2A, Table 2). Typical rosettes usually with distorted a central stem, with many

Table 1. Summary of measurements, and counts for type specimens of Lipotrapeza littoralis sp. nov. and L. japonica Heding and Panning, 1954.

\begin{tabular}{|c|c|c|c|c|c|c|c|c|c|c|}
\hline \multirow[b]{2}{*}{ Registration number $^{\mathrm{a}}$} & \multirow[b]{2}{*}{ Type status } & \multirow[b]{2}{*}{ Body state } & \multirow[b]{2}{*}{ Gonad state } & \multicolumn{2}{|c|}{ Body size $(\mathrm{mm})$} & \multicolumn{5}{|c|}{ Counts } \\
\hline & & & & Length & Width & Tentacles & $\begin{array}{c}\text { Anal } \\
\text { papillae }\end{array}$ & $\begin{array}{c}\text { Anal } \\
\text { scales }\end{array}$ & $\begin{array}{l}\text { Stone } \\
\text { canal }\end{array}$ & $\begin{array}{l}\text { Polian } \\
\text { vesicle }\end{array}$ \\
\hline \multicolumn{11}{|l|}{ L. littoralis sp. nov. } \\
\hline WMNH-INV-2013-13 & Paratype & relax & testis & 71 & 14 & 20 & 10 & 5 & 1 & 2 \\
\hline WMNH-INV-2014-46 & Paratype & contract & testis & 41 & 21 & 20 & 10 & 5 & 1 & 1 \\
\hline WMNH-INV-2014-67 & Holotype & relax & ovary & 77 & 19 & 20 & 10 & 5 & 1 & 2 \\
\hline WMNH-INV-2014-68 & Paratype & relax & ovary & 55 & 22 & 20 & 10 & 5 & 1 & 1 \\
\hline WMNH-INV-2014-69 & Paratype & eviscerating & ovary & 32 & 13 & 20 & 10 & 5 & 1 & 1 \\
\hline WMNH-INV-2014-101 & Paratype & relax & ovary & 77 & 17 & 20 & 10 & 5 & 1 & 2 \\
\hline \multicolumn{11}{|l|}{ L. japonica Heding and Panning, 1954} \\
\hline ZMUC-SYN-101 (large specimen) & Syntype & contract & ovary & 23 & 7 & 20 & $5^{\mathrm{b}}$ & $5^{\mathrm{b}}$ & 1 & 1 \\
\hline ZMUC-SYN-101 (small specimen) & Syntype & not dessected & not observed & 17 & 5 & not observed & 5 & 5 & \multicolumn{2}{|c|}{ not observed } \\
\hline
\end{tabular}

${ }^{a}$ WMNH: Wakayama Prefectural Museum of Natural History; ZMUC: Zoological Museum of University of Copenhagen. ${ }^{\mathrm{b}}$ Whole removed in the present syntype, and the counts are based on Heding and Panning (1954). 


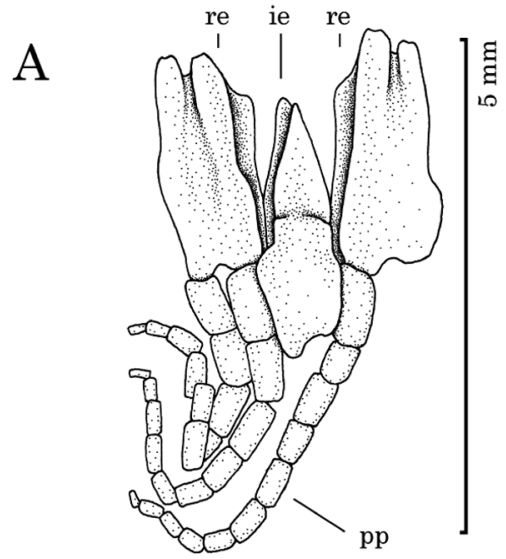

Calcareous ring
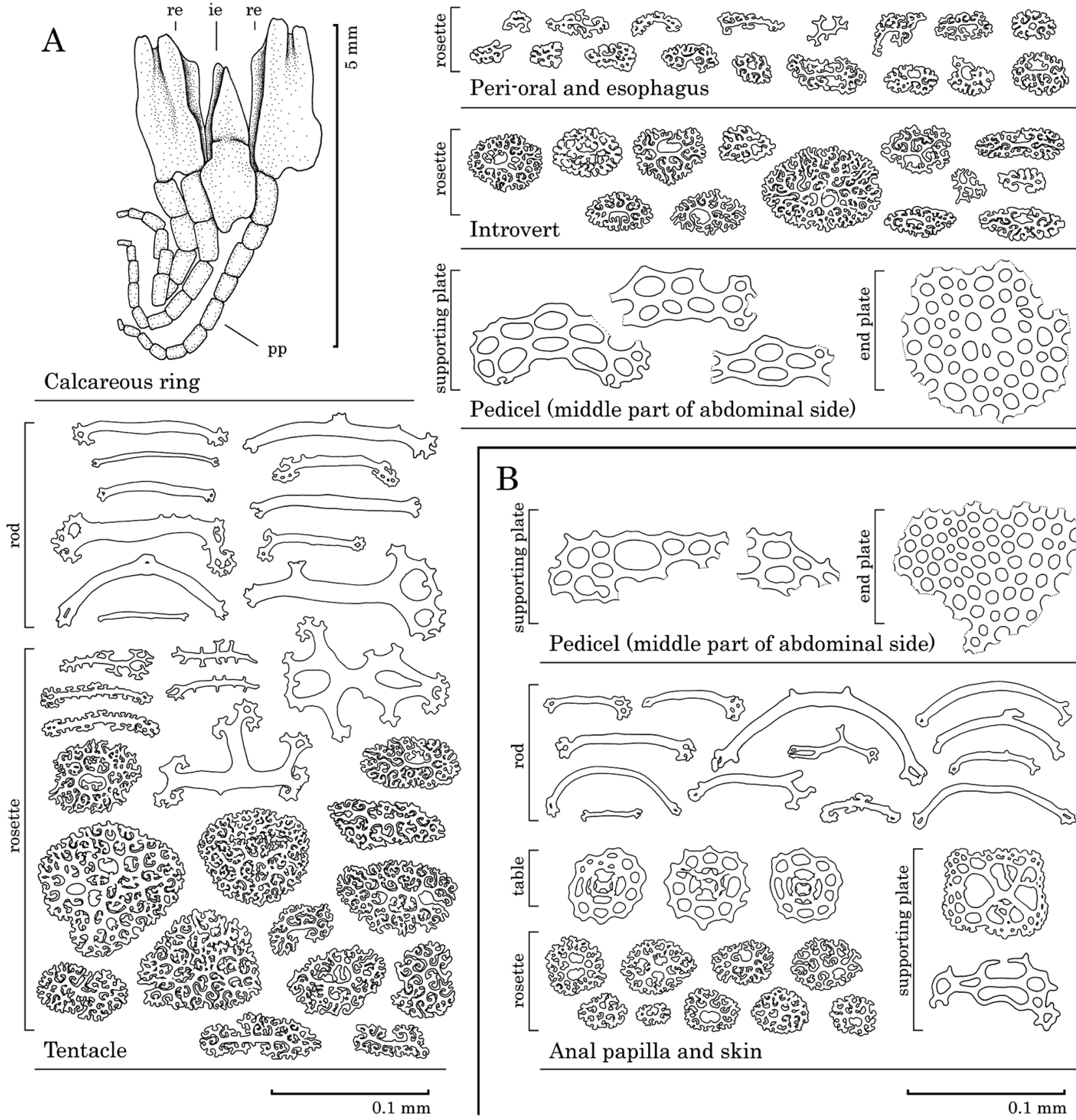

Pedicel (middle part of abdominal side)
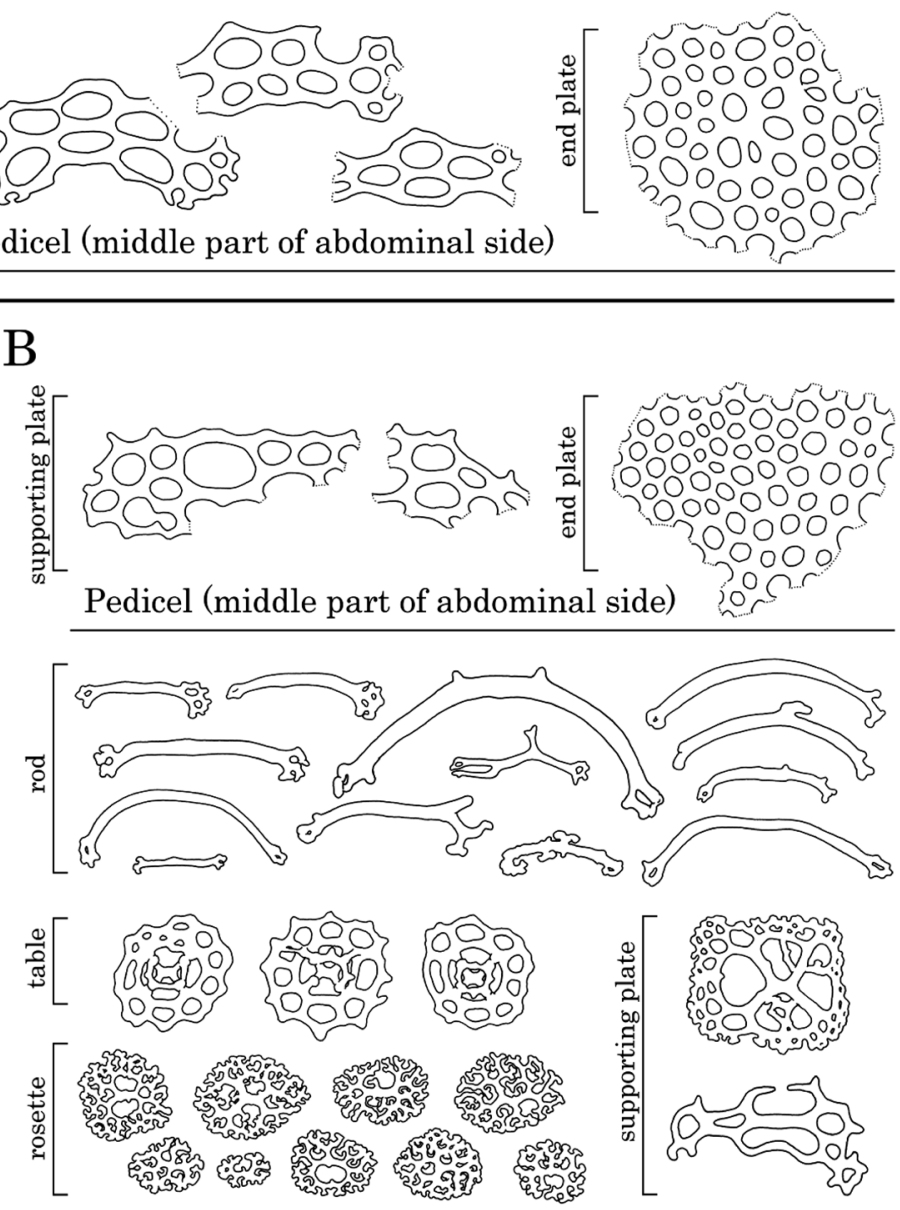

Anal papilla and skin

$0.1 \mathrm{~mm}$

Fig. 2. Elements of the calcareous ring, viewed from body cavity, Lipotrapeza japonica Heding and Panning, 1954, and ossicles of the mid-part of the inter-radial tentacle on the abdominal-lateral side; the peri-oral and esophageal skin on the abdominal-lateral side; the middle part of the skin of the introvert in the inter-radius, on the abdominal-lateral side; the pedicels of the abdominal side in the radius; appendage and skin around the anus on the abdominal-lateral side. A, ZMUC-SYN-101 large specimen (syntype); B, ZMUC-SYN-101 small specimen (syntype). Dotted line, eroded part.

free-ends of branches occurring in inner area as well as in marginal area; branches sometimes overlapping in inner area, resulting in rosettes with complicated shapes. This feature is relatively common in rosettes in the other tissues.

Because of contamination, ossicles from the peri-oral and esophageal processes were measured together, but the ossicles in the esophageal processes were relatively smaller than the peri-oral ossicles. Single type of ossicle found in introvert of large specimen, viz., "rosettes" $21-53 \mu \mathrm{m}$ long and $16-45 \mu \mathrm{m}$ wide (Fig. 2A, Table 2).

Integument of mid-abdominal body wall lacking ossicles in both large and small specimens. Two types of ossicles found in pedicel in mid-abdominal side in both large and small specimens, viz., "supporting plates" and "endplates." Both ossicle types present in both specimens; heavily erosion prevent accurate measurement (Fig. 2, 
Table 2. Measurements $(\mu \mathrm{m})$ of ossicles from tentacle, peri-oral skin, esophagus, and middle part of introvert of type specimens of Lipotrapeza littoralis sp. nov. and L. japonica Heding and Panning, 1954, on abdominal-lateral side.

\begin{tabular}{|c|c|c|c|c|c|c|c|c|c|c|c|c|c|c|c|}
\hline \multirow{3}{*}{ Registration number $^{\mathrm{a}}$} & \multicolumn{3}{|c|}{ Rods in tentacle } & \multicolumn{3}{|c|}{ Rosettes in tentacle } & \multicolumn{3}{|c|}{ Rosettes in peri-oral skin } & \multicolumn{3}{|c|}{ Rosettes in esophagus } & \multicolumn{3}{|c|}{ Rosettes in introvert } \\
\hline & & Length & Width $^{\mathrm{b}}$ & \multirow[b]{2}{*}{ (n } & Length & Width $^{\mathrm{c}}$ & \multirow[b]{2}{*}{$(r$} & Length & Width $^{\mathrm{c}}$ & \multirow[b]{2}{*}{$(n)$} & Length & Width $^{\mathrm{c}}$ & \multirow[b]{2}{*}{ (n) } & Length & Width $^{\mathrm{c}}$ \\
\hline & & $\begin{array}{c}\text { Mean } \pm \text { sd } \\
\text { (Range) }\end{array}$ & $\begin{array}{c}\text { Mean } \pm \text { sd } \\
\text { (Range) }\end{array}$ & & $\begin{array}{l}\text { Mean } \pm \text { sd } \\
\text { (Range) }\end{array}$ & $\begin{array}{l}\text { Mean } \pm \text { sd } \\
\text { (Range) }\end{array}$ & & $\begin{array}{c}\text { Mean } \pm \text { sd } \\
\text { (Range) }\end{array}$ & $\begin{array}{c}\text { Mean } \pm \text { sd } \\
\text { (Range) }\end{array}$ & & $\begin{array}{l}\text { Mean } \pm \text { sd } \\
\text { (Range) }\end{array}$ & $\begin{array}{l}\text { Mean } \pm \text { sd } \\
\text { (Range) }\end{array}$ & & $\begin{array}{l}\text { Mean } \pm \text { sd } \\
\text { (Range) }\end{array}$ & $\begin{array}{c}\text { Mean } \pm \text { sd } \\
\text { (Range) }\end{array}$ \\
\hline \multicolumn{16}{|l|}{ littoralis sp. nov. } \\
\hline WMN & (49) & $\begin{array}{c}61.8 \pm 12.8 \\
(41-92)\end{array}$ & $\begin{array}{c}2.9 \pm 1.0 \\
(2-7)\end{array}$ & $(51)$ & $\begin{array}{c}60.0 \pm 32.4 \\
(25-213)\end{array}$ & $\begin{array}{c}30.9 \pm 18.9 \\
(9-104)\end{array}$ & (47) & $\begin{array}{c}45.8 \pm 8.1 \\
(32-68)\end{array}$ & $\begin{array}{c}25.3 \pm 6.4 \\
(14-41)\end{array}$ & $(50)$ & $\begin{array}{c}36.1 \pm 8.1 \\
(22-64)\end{array}$ & $\begin{array}{c}22.8 \pm 5.3 \\
(10-38)\end{array}$ & (44) & $\begin{array}{l}17.1 \\
03)\end{array}$ & $\begin{array}{c}29.9 \pm 9.5 \\
(14-57)\end{array}$ \\
\hline WMNH & (37) & $\begin{array}{c}64.9 \pm 21.2 \\
(34-137)\end{array}$ & $\begin{array}{c}3.6 \pm 2.4 \\
(2-13)\end{array}$ & (63) & $\begin{array}{c}59.5 \pm 37.2 \\
(25-250)\end{array}$ & $\begin{array}{c}24.6 \pm 16.1 \\
(9-85)\end{array}$ & (59) & $\begin{array}{c}43.1 \pm 9.4 \\
(19-69)\end{array}$ & $\begin{array}{c}22.1 \pm 5.1 \\
(9-33)\end{array}$ & (58) & $\begin{array}{c}39.0 \pm 9.8 \\
(18-65)\end{array}$ & $\begin{array}{c}16.6 \pm 5.5 \\
(3-27)\end{array}$ & (59) & $\begin{array}{c}46.1 \pm 10.0 \\
(26-73)\end{array}$ & $\begin{array}{c}32.1 \pm 8.8 \\
(12-68)\end{array}$ \\
\hline WMN & (27) & $\begin{array}{c}64.3 \pm 13.0 \\
(35-93)\end{array}$ & $\begin{array}{c}2.9 \pm 1.2 \\
(2-8)\end{array}$ & (32) & $\begin{array}{c}103.7 \pm 53.1 \\
(35-254)\end{array}$ & $\begin{array}{c}65.7 \pm 43.4 \\
(19-203)\end{array}$ & (57) & $\begin{array}{c}39.0 \pm 8.8 \\
(25-63)\end{array}$ & $\begin{array}{c}27.0 \pm 5.4 \\
(15-45)\end{array}$ & $(60)$ & $\begin{array}{c}39.5 \pm 9.8 \\
(23-71)\end{array}$ & $\begin{array}{c}19.6 \pm 6.9 \\
(3-33)\end{array}$ & (33) & $\begin{array}{c}62.3 \pm 15.0 \\
(35-91)\end{array}$ & $\begin{array}{c}48.5 \pm 12.5 \\
(25-79)\end{array}$ \\
\hline WM & (34) & $\begin{array}{c}58.0 \pm 12.4 \\
(36-94)\end{array}$ & $\begin{array}{c}3.2 \pm 0.9 \\
(2-6)\end{array}$ & (66) & $\begin{array}{c}70.2 \pm 19.7 \\
(37-164)\end{array}$ & $\begin{array}{c}40.6 \pm 14.2 \\
(14-79)\end{array}$ & (39) & $\begin{array}{c}63.3 \pm 11.4 \\
(43-90)\end{array}$ & $\begin{array}{c}41.3 \pm 8.5 \\
(23-64)\end{array}$ & $(60)$ & $\begin{array}{c}38.8 \pm 9.2 \\
(19-78)\end{array}$ & $\begin{array}{c}17.5 \pm 5.0 \\
(3-29)\end{array}$ & (54) & $\begin{array}{c}64.9 \pm 12.7 \\
(34-107)\end{array}$ & $\begin{array}{c}40.1 \pm 9.8 \\
(21-61)\end{array}$ \\
\hline WMNH & (43) & $\begin{array}{c}68.0 \pm 16.7 \\
(42-129)\end{array}$ & $\begin{array}{c}3.9 \pm 1.4 \\
(2-10)\end{array}$ & (36) & $\begin{array}{c}84.0 \pm 46.8 \\
(27-241)\end{array}$ & $\begin{array}{c}40.3 \pm 12.6 \\
(16-75)\end{array}$ & (58) & $\begin{array}{c}57.6 \pm 20.4 \\
(20-101)\end{array}$ & $\begin{array}{c}31.5 \pm 11.8 \\
(15-71)\end{array}$ & (54) & $\begin{array}{c}39.0 \pm 10.5 \\
(21-73)\end{array}$ & $\begin{array}{c}16.9 \pm 6.8 \\
(3-29)\end{array}$ & (52) & $\begin{array}{c}63.8 \pm 18.7 \\
(29-127)\end{array}$ & $\begin{array}{c}34.8 \pm 13.6 \\
(15-73)\end{array}$ \\
\hline WMNH & $(52)$ & $\begin{array}{c}76.0 \pm 34.1 \\
(28-209)\end{array}$ & $\begin{array}{l}4.2 \pm 2.3 \\
(2-12)\end{array}$ & (48) & $\begin{array}{c}83.3 \pm 46.8 \\
(31-253)\end{array}$ & $\begin{array}{c}32.7 \pm 23.0 \\
(13-120)\end{array}$ & (66) & $\begin{array}{c}49.2 \pm 11.4 \\
(18-77)\end{array}$ & $\begin{array}{c}31.6 \pm 8.8 \\
(15-54)\end{array}$ & (56) & $\begin{array}{c}36.4 \pm 8.1 \\
(15-59)\end{array}$ & $\begin{array}{c}21.1 \pm 4.9 \\
(11-35)\end{array}$ & (61) & $\begin{array}{c}59.6 \pm 13.8 \\
(31-91)\end{array}$ & $\begin{array}{c}43.9 \pm 10.8 \\
(23-71)\end{array}$ \\
\hline \multicolumn{16}{|c|}{ L. japonica Heding and Panning, 1954} \\
\hline $\begin{array}{l}\text { ZMUC-SYN-101 } \\
\quad \text { (large specimen) }\end{array}$ & $(30)$ & $\begin{array}{c}84.0 \pm 25.1 \\
(33-156)\end{array}$ & $\begin{array}{l}5.9 \pm 2.5 \\
(2-13)\end{array}$ & (33) & $\begin{array}{c}66.2 \pm 18.8 \\
(33-119)\end{array}$ & $\begin{array}{c}37.4 \pm 17.3 \\
(13-84)\end{array}$ & $(44)^{d}$ & $\begin{array}{c}32.9 \pm 8.7 \\
(11-51)\end{array}$ & $\begin{array}{c}19.1 \pm 5.0 \\
(8-28)\end{array}$ & $($ ( ) & mixed in pe & ri-oral skin & (41) & $\begin{array}{c}41.9 \pm 7.8 \\
(21-53)\end{array}$ & $\begin{array}{c}26.4 \pm 5.0 \\
(16-45)\end{array}$ \\
\hline $\begin{array}{l}\text { ZMUC-SYN-101 } \\
\text { (small specimen) }\end{array}$ & $(一)$ & \multicolumn{2}{|c|}{ not observed } & $(-)$ & \multicolumn{2}{|c|}{ not observed } & $(-)$ & \multicolumn{2}{|c|}{ not observed } & $(一)$ & \multicolumn{2}{|c|}{ not observed } & $(-)$ & \multicolumn{2}{|c|}{ not observed } \\
\hline
\end{tabular}

${ }^{a}$ WMNH: Wakayama Prefectural Museum of Natural History; ZMUC: Zoological Museum of University of Copenhagen. ${ }^{b}$ Width of stem in the central part, not the maximam value.

${ }^{\mathrm{c}}$ Width in the broadest part. ${ }^{\mathrm{d}}$ Rosettes in oesophagus were measured together.

Table 3. Measurements $(\mu \mathrm{m})$ of ossicles from pedicels of type specimens of Lipotrapeza littoralis sp. nov. and L. japonica Heding and Panning, 1954, on abdominal side.

\begin{tabular}{|c|c|c|c|c|c|c|c|c|c|c|c|c|c|c|c|c|c|c|}
\hline \multirow{4}{*}{ Registration number ${ }^{\mathrm{a}}$} & \multicolumn{9}{|c|}{ Endplates in pedicels } & \multicolumn{9}{|c|}{ Supporting plates in pedicels } \\
\hline & \multicolumn{3}{|c|}{ Anterior part } & \multicolumn{3}{|c|}{ Middle part } & \multicolumn{3}{|c|}{ Posterior part } & \multicolumn{3}{|c|}{ Anterior part } & \multicolumn{3}{|c|}{ Middle part } & \multicolumn{3}{|c|}{ Posterior part } \\
\hline & \multirow[b]{2}{*}{$(n)$} & Major axis & No. holes ${ }^{\mathrm{b}}$ & \multirow[b]{2}{*}{$(n)$} & Major axis & No. holes ${ }^{b}$ & \multirow[b]{2}{*}{ (n) } & Major axis & No. holes ${ }^{\mathrm{b}}$ & \multirow[b]{2}{*}{$(n)$} & Length & Width $^{\mathrm{c}}$ & \multirow[b]{2}{*}{ (n) } & Length & Width $^{\mathrm{c}}$ & \multirow[b]{2}{*}{$(n)$} & Length & Width $^{\mathrm{c}}$ \\
\hline & & $\begin{array}{c}\text { Mean } \pm \text { sd } \\
\text { (Range) }\end{array}$ & $\begin{array}{l}\text { Mean } \pm \text { sd } \\
\text { (Range) }\end{array}$ & & $\begin{array}{c}\text { Mean } \pm \text { sd } \\
\text { (Range) }\end{array}$ & $\begin{array}{c}\text { Mean } \pm \text { sd } \\
\text { (Range) }\end{array}$ & & $\begin{array}{c}\text { Mean } \pm \text { sd } \\
\text { (Range) }\end{array}$ & $\begin{array}{c}\text { Mean } \pm \text { sd } \\
\text { (Range) }\end{array}$ & & $\begin{array}{c}\text { Mean } \pm \text { sd } \\
\text { (Range) }\end{array}$ & $\begin{array}{c}\text { Mean } \pm s d \\
\text { (Range) }\end{array}$ & & $\begin{array}{c}\text { Mean } \pm \text { sd } \\
\text { (Range) }\end{array}$ & $\begin{array}{c}\text { Mean } \pm \text { sd } \\
\text { (Range) }\end{array}$ & & $\begin{array}{l}\text { Mean } \pm \text { sd } \\
\text { (Range) }\end{array}$ & $\begin{array}{c}\text { Mean } \pm \text { sd } \\
\text { (Range) }\end{array}$ \\
\hline \multicolumn{19}{|l|}{ L. littoralis sp. nov. } \\
\hline WMNH-INV-2013-13 & $(8)$ & $\begin{array}{c}319 \pm 15 \\
(296-334)\end{array}$ & $\begin{array}{c}249 \pm 31 \\
(219-314)\end{array}$ & (6) & $\begin{array}{c}405 \pm 24 \\
(378-442)\end{array}$ & $\begin{array}{c}379 \pm 52 \\
(310-440)\end{array}$ & (12) & $\begin{array}{c}215 \pm 73 \\
(113-321)\end{array}$ & $\begin{array}{l}153 \pm 74 \\
(34-222)\end{array}$ & (19) & $\begin{array}{c}141 \pm 14 \\
(112-174)\end{array}$ & $\begin{array}{c}59 \pm 8 \\
(46-80)\end{array}$ & $(22)$ & $\begin{array}{c}175 \pm 21 \\
(146-208)\end{array}$ & $\begin{array}{c}47 \pm 11 \\
(12-66)\end{array}$ & (12) & $\begin{array}{c}142 \pm 24 \\
(102-179)\end{array}$ & $\begin{array}{c}38 \pm 7 \\
(24-48)\end{array}$ \\
\hline WMNH-INV-2014-46 & (8) & $\begin{array}{c}306 \pm 56 \\
(201-381)\end{array}$ & $\begin{array}{c}267 \pm 90 \\
(132-380)\end{array}$ & (6) & $\begin{array}{c}435 \pm 21 \\
(397-457)\end{array}$ & $\begin{array}{c}533 \pm 59 \\
(477-618)\end{array}$ & (8) & $\begin{array}{c}292 \pm 65 \\
(183-382)\end{array}$ & $\begin{array}{c}280 \pm 132 \\
(121-542)\end{array}$ & (24) & $\begin{array}{l}135 \pm 33 \\
(42-177)\end{array}$ & $\begin{array}{c}44 \pm 8 \\
(27-58)\end{array}$ & (23) & $\begin{array}{c}176 \pm 31 \\
(118-252)\end{array}$ & $\begin{array}{c}48 \pm 9 \\
(25-63)\end{array}$ & (23) & $\begin{array}{c}139 \pm 15 \\
(114-170)\end{array}$ & $\begin{array}{l}47 \pm 10 \\
(22-60)\end{array}$ \\
\hline WMNH-INV-2014-67 & (6) & $\begin{array}{c}229 \pm 56 \\
(175-297)\end{array}$ & $\begin{array}{c}140 \pm 63 \\
(75-216)\end{array}$ & (5) & $\begin{array}{c}395 \pm 26 \\
(351-415)\end{array}$ & $\begin{array}{c}371 \pm 65 \\
(320-446)\end{array}$ & (6) & $\begin{array}{c}216 \pm 19 \\
(193-246)\end{array}$ & $\begin{array}{l}114 \pm 20 \\
(90-145)\end{array}$ & $(21)$ & $\begin{array}{l}157 \pm 25 \\
(95-197)\end{array}$ & $\begin{array}{l}65 \pm 14 \\
(40-94)\end{array}$ & (7) & $\begin{array}{c}190 \pm 38 \\
(128-244)\end{array}$ & $\begin{array}{c}52 \pm 9 \\
(42-64)\end{array}$ & (17) & $\begin{array}{c}152 \pm 22 \\
(120-190)\end{array}$ & $\begin{array}{c}51 \pm 9 \\
(37-71)\end{array}$ \\
\hline WMNH-INV-2014-68 & (5) & $\begin{array}{c}256 \pm 56 \\
(197-314)\end{array}$ & $\begin{array}{c}219 \pm 90 \\
(112-310)\end{array}$ & (5) & $\begin{array}{c}411 \pm 60 \\
(317-468)\end{array}$ & $\begin{array}{l}430 \pm 101 \\
(290-538)\end{array}$ & (6) & $\begin{array}{c}264 \pm 45 \\
(209-328)\end{array}$ & $\begin{array}{c}247 \pm 83 \\
(154-402)\end{array}$ & (17) & $\begin{array}{c}161 \pm 18 \\
(129-205)\end{array}$ & $\begin{array}{l}60 \pm 14 \\
(34-89)\end{array}$ & (9) & $\begin{array}{c}162 \pm 13 \\
(141-182)\end{array}$ & $\begin{array}{c}58 \pm 10 \\
(33-67)\end{array}$ & $(20)$ & $\begin{array}{c}160 \pm 24 \\
(119-209)\end{array}$ & $\begin{array}{c}59 \pm 9 \\
(44-71)\end{array}$ \\
\hline WMNH-INV-2014-69 & $(8)$ & $\begin{array}{c}249 \pm 84 \\
(114-325)\end{array}$ & $\begin{array}{l}216 \pm 110 \\
(250-342)\end{array}$ & (5) & $\begin{array}{c}286 \pm 51 \\
(209-346)\end{array}$ & $\begin{array}{c}260 \pm 84 \\
(134-370)\end{array}$ & (7) & $\begin{array}{c}244 \pm 69 \\
(161-311)\end{array}$ & $\begin{array}{c}197 \pm 88 \\
(100-286)\end{array}$ & (24) & $\begin{array}{l}133 \pm 18 \\
(92-163)\end{array}$ & $\begin{array}{l}47 \pm 17 \\
(13-85)\end{array}$ & (13) & $\begin{array}{c}142 \pm 27 \\
(107-210)\end{array}$ & $\begin{array}{l}56 \pm 11 \\
(33-69)\end{array}$ & (15) & $\begin{array}{c}126 \pm 10 \\
(109-151)\end{array}$ & $\begin{array}{l}50 \pm 10 \\
(38-77)\end{array}$ \\
\hline WMNH-INV-2014-101 & (6) & $\begin{array}{c}279 \pm 43 \\
(214-334)\end{array}$ & $\begin{array}{c}261 \pm 91 \\
(148-368)\end{array}$ & (5) & $\begin{array}{c}361 \pm 46 \\
(310-412)\end{array}$ & $\begin{array}{l}403 \pm 115 \\
(306-590)\end{array}$ & (7) & $\begin{array}{c}216 \pm 64 \\
(133-301)\end{array}$ & $\begin{array}{c}153 \pm 73 \\
(59-278)\end{array}$ & (9) & $\begin{array}{c}155 \pm 23 \\
(114-189)\end{array}$ & $\begin{array}{c}50 \pm 8 \\
(36-65)\end{array}$ & (6) & $\begin{array}{c}163 \pm 14 \\
(150-188)\end{array}$ & $\begin{array}{c}45 \pm 8 \\
(39-61)\end{array}$ & (6) & $\begin{array}{c}124 \pm 14 \\
(102-140)\end{array}$ & $\begin{array}{c}50 \pm 6 \\
(43-56)\end{array}$ \\
\hline \multicolumn{19}{|c|}{ L. japonica Heding and Panning, 1954} \\
\hline $\begin{array}{l}\text { ZMUC-SYN-101 } \\
\quad \text { (large specimen) }\end{array}$ & $(-)$ & \multicolumn{2}{|c|}{ not observed } & $(1)^{\mathrm{d}}$ & 120 & 60 & $(-)$ & \multicolumn{2}{|c|}{ not observed } & $(-)$ & \multicolumn{2}{|c|}{ not observed } & $(3)^{\mathrm{d}}$ & $\begin{array}{l}113 \pm 21 \\
(90-130)\end{array}$ & $\begin{array}{c}37 \pm 2 \\
(35-39)\end{array}$ & $(-)$ & \multicolumn{2}{|c|}{ not observed } \\
\hline $\begin{array}{l}\text { ZMUC-SYN-101 } \\
\quad \text { (small specimen) }\end{array}$ & $(-)$ & not obs & served & $(1)^{\mathrm{d}}$ & 220 & 240 & $(-)$ & not obs & served & $(-)$ & not obs & served & $(3)^{\mathrm{d}}$ & $\begin{array}{l}101 \pm 28 \\
(81-133)\end{array}$ & $\begin{array}{c}42 \pm 4 \\
(38-46)\end{array}$ & $(-)$ & not obs & served \\
\hline
\end{tabular}

a WMNH: Wakayama Prefectural Museum of Natural History; ZMUC: Zoological Museum of University of Copenhagen. ${ }^{\mathrm{b}}$ Exhaustive count or, in case of broken endplates, estimated by doubling count of half of plate or putting togather the fragments. ${ }^{\mathrm{c}}$ Width in the central part, not in the broadest part. ${ }^{\mathrm{d}}$ The ossicles were eroded by a long period storage, therefore we could not measure them accurately, and the values estimated from the remaining parts or fragments.

Table 3).

Four types of ossicles found in papilla and skin around anus of small specimen, viz., "rosettes" 23-64 $\mu \mathrm{m}$ long and
15-53 $\mu \mathrm{m}$ wide; "rods" 39-136 $\mu \mathrm{m}$ long and 3-10 $\mu \mathrm{m}$ wide at center of stem; "tables" with low 4-pillared spire and one cross-beam, and with 9-11 marginal holes in disc, disc 
Table 4. Measurements ( $\mu \mathrm{m})$ of ossicles from anal papilla of type specimens of Lipotrapeza littoralis sp. nov. and L. japonica Heding and Panning, 1954, on abdominal-lateral side.

\begin{tabular}{|c|c|c|c|c|c|c|c|c|c|c|c|c|}
\hline \multirow{3}{*}{ Registration number ${ }^{a}$} & \multicolumn{3}{|c|}{ Rods } & \multicolumn{3}{|c|}{ Rosettes } & \multicolumn{3}{|c|}{ Supporting plates } & \multicolumn{3}{|c|}{ Tables } \\
\hline & \multirow[b]{2}{*}{$(n)$} & Length & Width $^{\mathrm{b}}$ & \multirow[b]{2}{*}{ (n) } & Length & Width $^{\mathrm{c}}$ & \multirow[b]{2}{*}{$(n)$} & Length & Width $^{\mathrm{d}}$ & \multirow[b]{2}{*}{$(n)$} & Major axis ${ }^{\mathrm{e}}$ & No. holes ${ }^{f}$ \\
\hline & & $\begin{array}{l}\text { Mean } \pm \text { sd } \\
\text { (Range) }\end{array}$ & $\begin{array}{l}\text { Mean } \pm \text { sd } \\
\text { (Range) }\end{array}$ & & $\begin{array}{l}\text { Mean } \pm \text { sd } \\
\text { (Range) }\end{array}$ & $\begin{array}{l}\text { Mean } \pm \text { sd } \\
\text { (Range) }\end{array}$ & & $\begin{array}{l}\text { Mean } \pm \text { sd } \\
\text { (Range) }\end{array}$ & $\begin{array}{l}\text { Mean } \pm \text { sd } \\
\text { (Range) }\end{array}$ & & $\begin{array}{l}\text { Mean } \pm \text { sd } \\
\text { (Range) }\end{array}$ & $\begin{array}{c}\text { Mean } \pm \text { sd } \\
\text { (Range) }\end{array}$ \\
\hline \multicolumn{13}{|l|}{ L. littoralis sp. nov. } \\
\hline WMNH-INV-2013-13 & $(18)$ & $\begin{array}{c}74.1 \pm 18.9 \\
(30-112)\end{array}$ & $\begin{array}{l}5.3 \pm 1.5 \\
(2-8)\end{array}$ & (34) & $\begin{array}{c}44.8 \pm 12.3 \\
(26-88)\end{array}$ & $\begin{array}{l}35.2 \pm 10.1 \\
(16-70)\end{array}$ & $(16)$ & $\begin{array}{c}114.3 \pm 28.7 \\
(66-178)\end{array}$ & $\begin{array}{l}56.1 \pm 18.1 \\
(30-94)\end{array}$ & $(6)$ & $\begin{array}{c}67.0 \pm 3.7 \\
(61-70)\end{array}$ & $\begin{array}{c}9.7 \pm 5.1 \\
(3-17)\end{array}$ \\
\hline WMNH-INV-2014-46 & $(30)$ & $\begin{array}{c}68.3 \pm 9.6 \\
(53-94)\end{array}$ & $\begin{array}{c}4.1 \pm 1.2 \\
(2-8)\end{array}$ & $(23)$ & $\begin{array}{c}45.9 \pm 12.5 \\
(24-81)\end{array}$ & $\begin{array}{c}24.3 \pm 10.7 \\
(14-61)\end{array}$ & $(18)$ & $\begin{array}{c}115.1 \pm 28.3 \\
(55-160)\end{array}$ & $\begin{array}{c}57.6 \pm 28.5 \\
(20-141)\end{array}$ & $(6)$ & $\begin{array}{l}62.8 \pm 7.5 \\
(53-75)\end{array}$ & $\begin{array}{c}7.7 \pm 2.7 \\
(5-11)\end{array}$ \\
\hline WMNH-INV-2014-67 & (5) & $\begin{array}{c}61.6 \pm 8.0 \\
(51-71)\end{array}$ & $\begin{array}{c}4.4 \pm 1.1 \\
(3-6)\end{array}$ & $(27)$ & $\begin{array}{c}47.6 \pm 13.4 \\
(26-69)\end{array}$ & $\begin{array}{c}37.6 \pm 12.7 \\
(15-57)\end{array}$ & $(12)$ & $\begin{array}{l}93.3 \pm 16.4 \\
(71-127)\end{array}$ & $\begin{array}{l}44.0 \pm 10.1 \\
(27-59)\end{array}$ & $(6)$ & $\begin{array}{l}75.0 \pm 6.0 \\
(66-82)\end{array}$ & $\begin{array}{c}15.3 \pm 2.3 \\
(12-18)\end{array}$ \\
\hline WMNH-INV-2014-68 & $(12)$ & $\begin{array}{c}64.2 \pm 15.2 \\
(39-87)\end{array}$ & $\begin{array}{c}4.3 \pm 0.8 \\
(3-6)\end{array}$ & $(26)$ & $\begin{array}{l}45.2 \pm 11.0 \\
(31-77)\end{array}$ & $\begin{array}{c}30.5 \pm 8.4 \\
(16-54)\end{array}$ & $(18)$ & $\begin{array}{c}100.9 \pm 22.3 \\
(69-153)\end{array}$ & $\begin{array}{c}58.4 \pm 25.3 \\
(15-112)\end{array}$ & $(8)$ & $\begin{array}{c}67.6 \pm 8.9 \\
(53-81)\end{array}$ & $\begin{array}{c}10.9 \pm 3.5 \\
(6-16)\end{array}$ \\
\hline WMNH-INV-2014-69 & (11) & $\begin{array}{c}56.5 \pm 8.9 \\
(44-68)\end{array}$ & $\begin{array}{c}3.7 \pm 1.1 \\
(2-6)\end{array}$ & (17) & $\begin{array}{c}50.2 \pm 6.4 \\
(38-63)\end{array}$ & $\begin{array}{c}41.7 \pm 8.7 \\
(23-55)\end{array}$ & $(15)$ & $\begin{array}{c}90.7 \pm 20.3 \\
(58-131)\end{array}$ & $\begin{array}{c}40.4 \pm 14.3 \\
(22-71)\end{array}$ & (13) & $\begin{array}{c}59.9 \pm 11.3 \\
(48-94)\end{array}$ & $\begin{array}{c}13.0 \pm 4.6 \\
(6-20)\end{array}$ \\
\hline WMNH-INV-2014-101 & (14) & $\begin{array}{c}58.7 \pm 12.2 \\
(43-81)\end{array}$ & $\begin{array}{l}4.4 \pm 1.0 \\
(3-6)\end{array}$ & $(35)$ & $\begin{array}{l}49.2 \pm 13.1 \\
(26-81)\end{array}$ & $\begin{array}{l}36.1 \pm 13.0 \\
(14-67)\end{array}$ & $(16)$ & $\begin{array}{c}112.4 \pm 23.0 \\
(63-140)\end{array}$ & $\begin{array}{l}51.4 \pm 16.7 \\
(35-97)\end{array}$ & $(11)$ & $\begin{array}{c}75.3 \pm 13.4 \\
(55-94)\end{array}$ & $\begin{array}{c}16.9 \pm 8.3 \\
(5-35)\end{array}$ \\
\hline \multicolumn{13}{|c|}{ L. japonica Heding and Panning, 1954} \\
\hline $\begin{array}{l}\text { ZMUC-SYN-101 } \\
\quad \text { (large specimen) }\end{array}$ & $(-)$ & \multicolumn{2}{|c|}{ not observed } & $(-)$ & \multicolumn{2}{|c|}{ not observed } & $(-)$ & \multicolumn{2}{|c|}{ not observed } & $(-)$ & \multicolumn{2}{|c|}{ not observed } \\
\hline $\begin{array}{l}\text { ZMUC-SYN-101 } \\
\text { (small specimen) }\end{array}$ & $(18)$ & $\begin{array}{c}78.3 \pm 24.5 \\
(39-136)\end{array}$ & $\begin{array}{c}5.4 \pm 1.6 \\
(3-10)\end{array}$ & $(16)$ & $\begin{array}{c}39.1 \pm 9.8 \\
(23-64)\end{array}$ & $\begin{array}{c}29.3 \pm 9.8 \\
(15-53)\end{array}$ & (2) & $\begin{array}{c}66.0 \\
(53-79)\end{array}$ & $\begin{array}{c}36.5 \\
(33-40)\end{array}$ & (3) & $\begin{array}{c}54.3 \pm 2.3 \\
(53-57)\end{array}$ & $\begin{array}{c}9.7 \pm 1.2 \\
(9-11)\end{array}$ \\
\hline
\end{tabular}

${ }^{a}$ WMNH: Wakayama Prefectural Museum of Natural History; ZMUC: Zoological Museum of University of Copenhagen. ${ }^{\mathrm{b}}$ Width of stem in the central part, not the maximam value. ${ }^{\mathrm{c}}$ Width in the broadest part. ${ }^{\mathrm{d}}$ Width in the central part, not in the broadest part. ${ }^{\mathrm{e}}$ Major axis (diameter) of the disc of table ossicle. ${ }^{\mathrm{f}}$ Number of the holes in the marginal area of disc of table ossicle.

53-57 $\mu \mathrm{m}$ long; and "supporting plates" 53-79 $\mu \mathrm{m}$ long and 33-40 $\mu \mathrm{m}$ wide, some of which are apparently related to the "table" (Fig. 2, Table 4). Simple-shaped rods observed near tip of anal papilla; complex-shaped rods, supporting plates, and rosettes observed in middle to basal part of anal papilla. Normal-shaped tables observed in basal part of anal papilla and peripheral anal skin.

Distribution. So far known only from the type locality, $110 \mathrm{~m}$ deep in the Genkainada Sea, Nagasaki Prefecture, western Japan.

Remarks. The morphological account provided by Heding and Panning (1954) was generally so precise and accurate that our observations yielded no taxonomic novelty. However, the general appearance of the body (Fig. 1) and the ossicle morphology (Fig. 2), especially the existence of the tables in the anal appendage, for which little information is available in the literature, may be useful for the identification of this species.

\section{Lipotrapeza littoralis $\mathrm{sp}$. nov.}

(Figs. 3; 4; 5; 6; 7)

Material examined. Holotype, WMNH-INV-2014-67 (ATA, anesthetized, length $77 \mathrm{~mm}$, width $19 \mathrm{~mm}$ ). Paratypes: WMNH-INV-2013-13 (BOJ, anesthetized, length $71 \mathrm{~mm}$, width $14 \mathrm{~mm}$ ); WMNH-INV-2014-46 (TOS, length $41 \mathrm{~mm}$, width $21 \mathrm{~mm}$ ); WMNH-INV-2014-68 (ATA, anesthetized, length $55 \mathrm{~mm}$, width $22 \mathrm{~mm}$ ); WMNH-
INV-2014-69 (ATA, length $32 \mathrm{~mm}$, width $13 \mathrm{~mm}$ ); WMNHINV-2014-101 (ATA, anesthetized, length $77 \mathrm{~mm}$, width $17 \mathrm{~mm}$ ); collection details given in Materials and Methods section above.

Other materials: WMNH-INV-2013-14 (BOJ); WMNHINV-2014-70-72 (ATA); WMNH-INV-2014-102-112 (ATA).

Description. Body curved or U-shaped (Fig. 3A), with both ends tapered and turned upwards; body wall soft and thick. Body (living and preserved) brown with whitish marbling; yellowish color occasionally observed.

Twenty tentacles arranged in a double circle $(15+5)$, including five pairs of large inter-radial tentacles and five single small radial tentacles in the outer circle and five very small radial tentacles in the inner circle (Fig. 4A). Long processes present, surrounding entrance of esophagus.

Retractable pedicels covering entire body at an even density. Color of pedicels (living and preserved) generally paler than adjacent body, tips white, rarely black. Pedicels becoming gradually smaller anterior and posterior to the middle portion of the body, absent in introvert region. On anterior body tip, pedicels of radius apparently larger than those of inter-radius.

Ten anal papillae and five anal scales in radii (five pairs of anal papillae in outer circle and five anal scales in inner circle; Fig. 4B, Table 1), color generally same as adjacent body skin. Each anal scale containing a long calcareous 

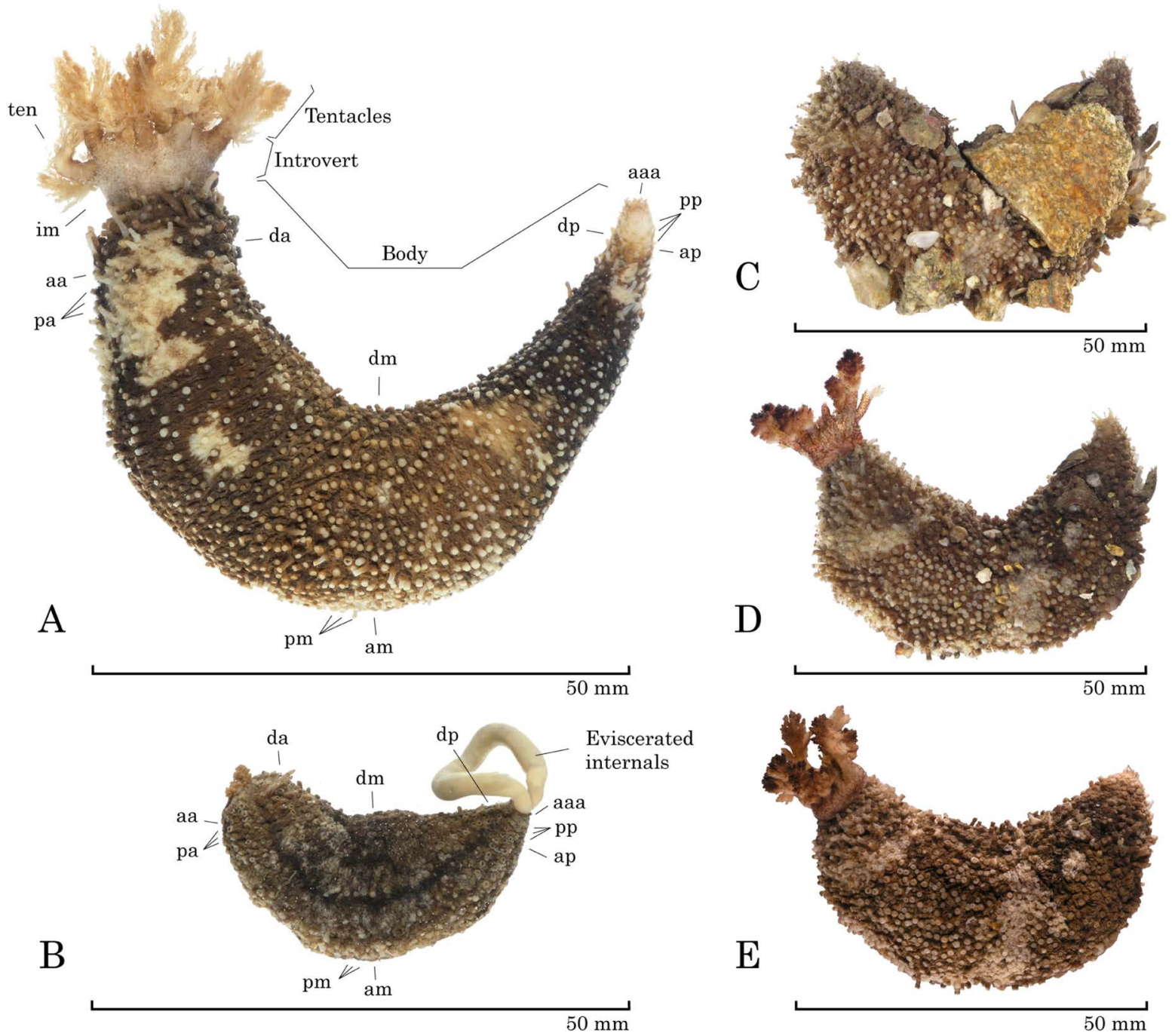

Fig. 3. Lateral views, Lipotrapeza littoralis sp. nov., A, WMNH-INV-2014-67 (holotype); B, WMNH-INV-2014-69 (paratype); C-E, WMNH-INV-2014-68 (paratype). A, E, fixed with anesthesia; B, fixed without anesthesia; C, D, live specimen with pebbles attached, before (C) and after (D) anesthetization. Abbreviations indicate sites of tissue sampling for ossicle examination: aaa, anal appendage and anal integument on abdominal-lateral side; aa, anterior part of body on abdominal side; am, middle part of body on abdominal side; ap, posterior part of body on abdominal side; da, anterior part of body on dorsal side; dm, middle part of body on dorsal side; dp, posterior part of body on dorsal side; im, middle part of introvert; pa, pedicels of anterior part; pm, pedicels of middle part; pp, pedicels of posterior part; ten, tentacle.

\section{structure.}

Long calcareous ring present in holotype, encircling esophagus (Fig. 5). Pieces of radial and inter-radial elements not forming a mosaic pattern. Main radial plates rectangular, notched anteriorly and posteriorly; main interradial plates sagittate, sharply protruding anteriorly. Radials with paired posterior prolongations, long but not discrete. Two Polian vesicles and one stone canal present in holotype (Table 1). Gonad tubules with small numbers of branches.

Two types of ossicles present in tentacles, viz., "rosettes" and "rods," as well as a full series of intermediate forms; rosettes 35-254 $\mu \mathrm{m}$ long and 19-203 $\mu \mathrm{m}$ wide; rods 35$93 \mu \mathrm{m}$ long and $2-8 \mu \mathrm{m}$ wide at center of the stem (in holotype) (Fig. 5, Table 2). Typical rosettes having relatively linear central stem, with free-ends of branches mostly restricted to marginal area, giving most rosettes simple shape resembling the plate ossicles. This feature relatively common in rosettes in the other tissues.

Single type of ossicle found in peri-oral skin, viz., "rosettes" $25-63 \mu \mathrm{m}$ long and $15-45 \mu \mathrm{m}$ wide (in holotype) (Fig. 5, Table 2). Two types of ossicles found in esophagus, viz., "small rosettes" and "rods," as well as a full series of intermediate forms; these ossicles, irrespective of form, 23-71 $\mu \mathrm{m}$ long and 3-33 $\mu \mathrm{m}$ wide (in holotype) (Fig. 5, Table 2). Single type of ossicle found in introvert, viz., "rosettes" 35-91 $\mu \mathrm{m}$ long and 25-79 $\mu \mathrm{m}$ wide (in holotype) (Fig. 5, Table 2).

Integument of body wall lacking ossicles anteriorly and posteriorly, on both abdominal and dorsal sides.

Two types of ossicles found in pedicels throughout anterior to posterior body, viz., "supporting plates" and "end- 

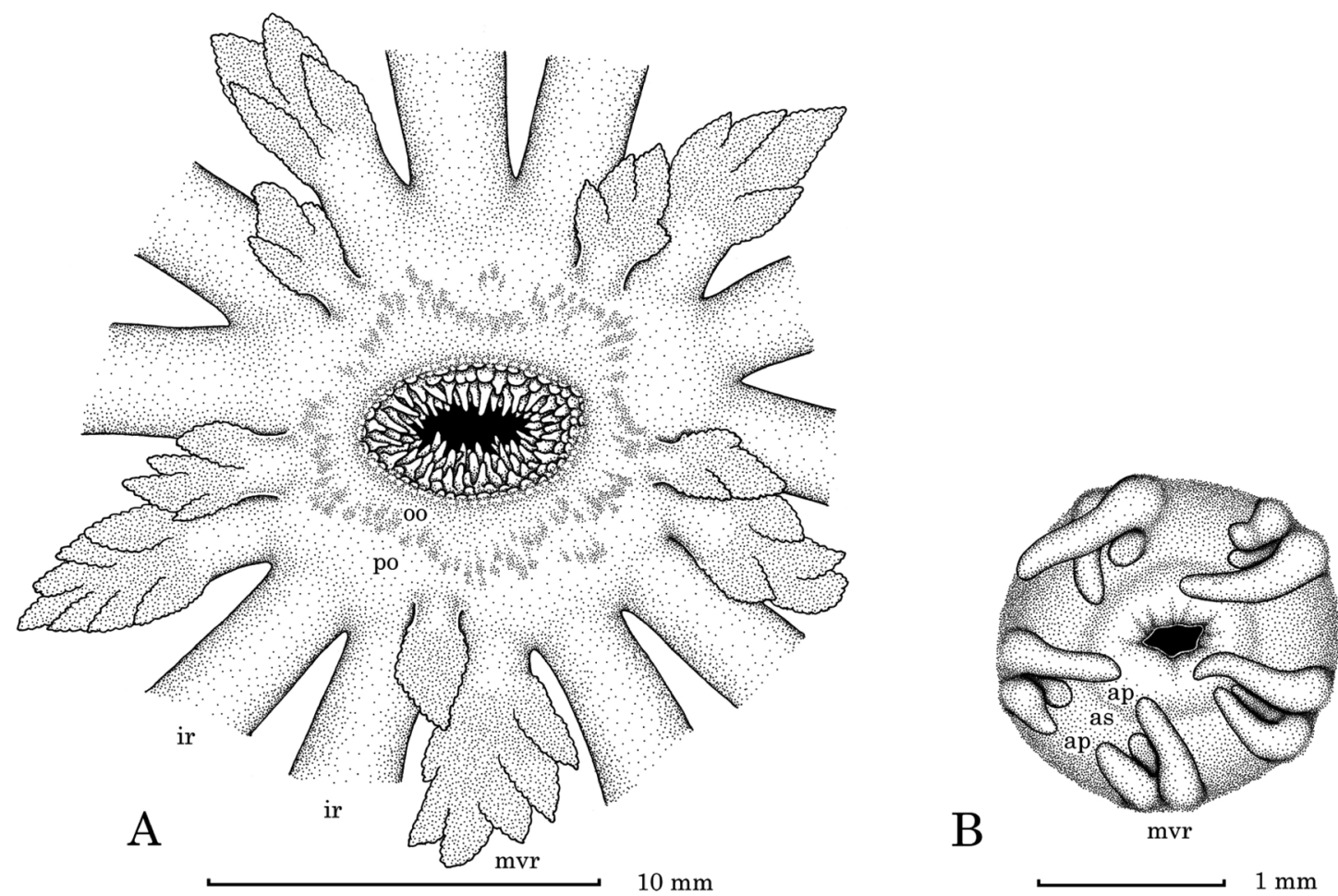

Fig. 4. Front view of oral and anal opening, Lipotrapeza littoralis sp. nov., WMNH-INV-2014-67 (holotype). A, oral opening; B, anal opening. Abbreviations: ap, anal papilla; as, anal scale; ir, inter-radial tentacle; mvr, mid-ventral radial part; oo, oral opening; po, peri-oral part.

plates." In middle part of abdominal side, supporting plates $128-244 \mu \mathrm{m}$ long and $42-64 \mu \mathrm{m}$ wide, and diameter of endplates $351-415 \mu \mathrm{m}$, with approximately $320-446$ holes (in holotype) (Fig. 5, Table 3).

Four types of ossicles found in anal papilla and skin around anus, viz., "rosettes" $26-69 \mu \mathrm{m}$ long and $15-57 \mu \mathrm{m}$ long; "rods" $51-71 \mu \mathrm{m}$ long and 3-6 $4 \mathrm{~m}$ wide at the center of the stem; "tables" with low 4-pillared spire and one cross-beam, diameter of disc 66-82 $\mu$ m with 12-18 marginal holes; and "supporting plates," some of this type apparently related to the "tables," with 71-127 $\mu \mathrm{m}$ long and $27-59 \mu \mathrm{m}$ wide (in holotype) (Fig. 5, Table 4). Simple-shaped rods observed in near tip of anal papilla, complex-shaped rods, supporting plates, and rosettes observed in middle to basal part of anal papilla. Normal-shaped tables observed in basal part of anal papilla and anal periphery skin.

Distribution. In sandy-gravel sediment under boulders in lower part of intertidal zone, coast of Nagasaki, Yamaguchi, and Wakayama Prefectures, western Japan. This species is probably distributed widely in other parts of Japan as well.

Ecology. Individuals were found burrowing in the sand and pebbles that filled up the spaces between boulders, often spreading their tentacles into the water. We found the following other species of sea cucumbers nearby: Apostichopus sp. (in ATA, BOJ, and TOS); Eupentacta sp. (in ATA and TOS); Pseudocnus pawsoni Won and Rho, 1998 (in ATA); Cladolabes kirara Yamana, Iwaoka and Hyakutake, 2014 (in BOJ); Holothuria (Lessonothuria) pardalis Selenka,
1867 (in BOJ); Afocucumis africana (Semper, 1867) (in BOJ and TOS); Stolus buccalis (Stimpson, 1855) (in BOJ); and Neothyonidium sp. (in TOS).

Etymology. The specific name alludes to its commonness in shallow to intertidal waters in western Japan.

Remarks. Appearance of anesthetized specimens not drastically altered by fixation, and general color of specimens also not seriously affected by fixation/preservation (Fig. 3C-E). Body (living and preserved) occasionally whitish-brown with brown marbling. Posterior body tip generally paler than adjacent body. Color of tentacles and introvert (living and preserved) quite variable; tentacle tips (nodules) generally tinged with black, other parts whitish-brown with brown variegation, occasionally colorless or wholly black. Pedicels covering entire body at an even density, but occasionally significantly sparse in middle inter-radii, with a narrow band of bare skin running from anterior to posterior (Fig. 3B). Stout short calcareous ring (Fig. 6A), or very long, thick calcareous ring (Fig. 6B) also present. Number of Polian vesicles one or two (Table 1).

Ossicle variations are found in this species very frequently (Fig. 7), and ossicle measurements also varied (Table 2-4). Furthermore, in the tentacles, some regenerated individuals frequently have enlarged and swollen rosettes and rods, which occur at a low frequency in most individuals. In this species, two types of ossicles are usually found in the pedicels throughout the anterior and posterior body, viz., "supporting plates"and "endplates," although another 

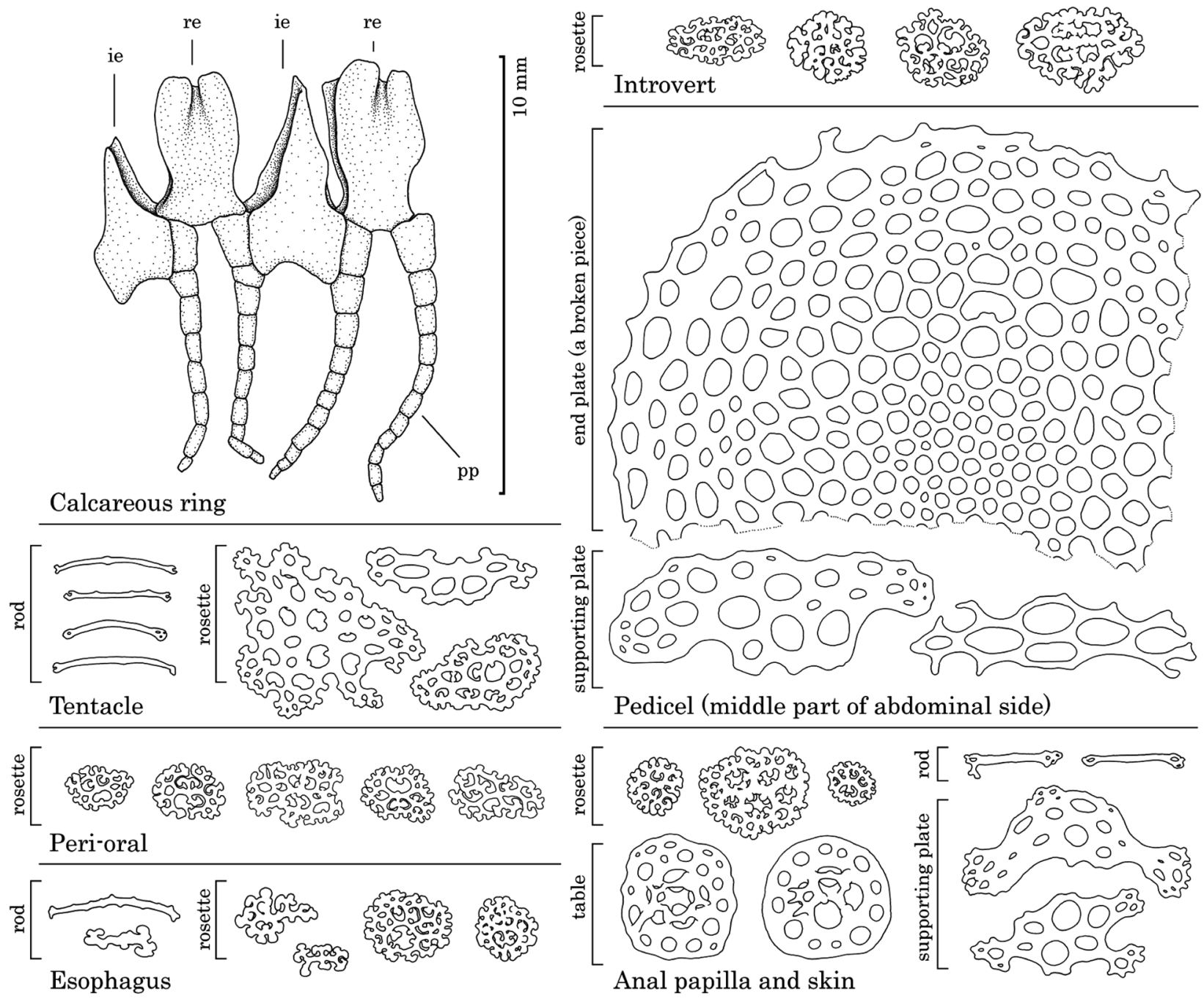

$0.1 \mathrm{~mm}$

Fig. 5. Elements of the calcareous ring, viewed from body cavity, and typical ossicles, Lipotrapeza littoralis sp. nov., WMNHINV-2014-67 (holotype); ossicles of the mid-part of the inter-radial tentacle on the abdominal-lateral side; the peri-oral and esophageal skin on the abdominal-lateral side; the middle part of the skin of the introvert in the inter-radius on the abdominal-lateral side; the pedicels of the abdominal side in the radius; appendage and skin around the anus on the abdominal-lateral side. Abbreviations: ie, inter-radial element; pp, posterior prolongation; re, radial element. Dotted line, missing.

type of ossicles was rarely observed $(n=3)$ in the smallest specimen (WMNH-INV-2014-69, paratype) in the basal part of the pedicel walls on the posterior body (not observed in adjacent body integument), viz., "tables" with a low spire and one cross-beam, and with irregularly shaped discs $63-79 \mu \mathrm{m}$ long (mean $\pm \mathrm{sd}=71.3 \pm 8.0)$ and $50-61 \mu \mathrm{m}$ wide (mean $\pm \mathrm{sd}=54.0 \pm 6.1$ ) (Fig. 7).

\section{Comparison of the rosette ossicles in the two species}

There was a significant positive relationship between the approximate area of the rosettes and the number of freeends of branches in the pooled data of 10 typical rosettes from five body regions (Fig. 8). The regressions were estimated as follows:

$$
N f=0.519 A^{0.564} \quad\left(n=300, \mathrm{r}^{2}=0.65, P<0.01\right)
$$

in Lipotrapeza littoralis sp. nov., and in L. japonica:

$$
N f=0.207 A^{0.808} \quad\left(n=40, \mathrm{r}^{2}=0.90, P<0.01\right)
$$

where $N f$ denotes the total number of free-ends of branches in a rosette, and $A$ denotes the approximate value of the area of a rosette (details given in Materials and Methods section). For statistical comparison, regressions (1) and (2) were transformed into logarithmic forms, $\left(1^{\prime}\right)$ and $\left(2^{\prime}\right)$, respectively, as follows:

$$
\begin{aligned}
& \log _{\mathrm{e}} N f=0.564 \log _{\mathrm{e}} A+\log _{\mathrm{e}} 0.519 \\
& \log _{\mathrm{e}} N f=0.808 \log _{\mathrm{e}} A+\log _{\mathrm{e}} 0.207
\end{aligned}
$$



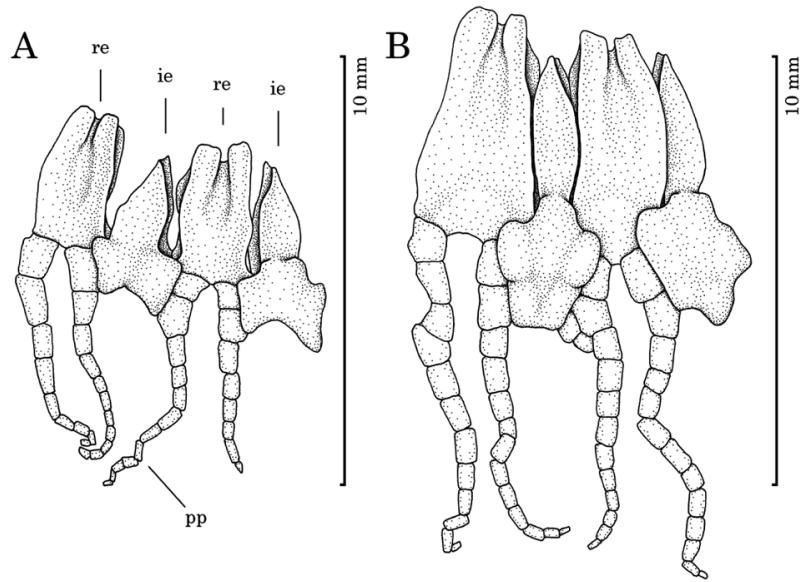

Fig. 6. Different forms of calcareous rings, Lipotrapeza littoralis sp. nov., viewed from body cavity. A, WMNH-INV-2014-101 (paratype); B, WMNH-INV-2013-13 (paratype).

In these forms, significant differences were observed between the two linear regressions (ANCOVA, slope: $P<0.01$, intercept: $P<0.01$ ). The density of the free-ends of branches is thus apparently higher in L. japonica, and the appearance of the rosettes in the two species is generally different.

\section{Discussion}

We were not able to find any table ossicles in the body integument of the two syntypes of Lipotrapeza japonica, as Heding and Panning (1954) had reported. On the other hand, we did find normal tables in the anal papilla of the small syntype of $L$. japonica, whereas Heding and Panning (1954) reported that they could find only rudimental tables and could not find normal tables in this structure. According to a morphological judgment made on the basis of the external body and ossicles, both specimens belong to the same species, and Heding and Panning (1954) produced an incorrect diagnosis.

Heding and Panning (1954) reported that Lipotrapeza japonica had 20 tentacles in triple circles $(10+5+5)$, but we observed a double circle $(15+5)$ in our present study. In terms of tentacle arrangement, we confirmed that Heding and Panning (1954) did not observe the actual arrangement of the tentacles (they had not dissected the oral part of the syntype, although the other parts of the body were carefully dissected). In fact, they described the tentacle arrangement based on observations of the tentacular canals, and this description was greatly affected by Ohshima's (1912) report, as they noted. Thus, their diagnosis of L. japonica mostly agreed with Clark's (1938) diagnosis, with the exception of the shape of the calcareous ring.

In the present study, we confirmed that the shape of the calcareous ring of the syntype of Lipotrapeza japonica agreed with the illustrated shape in Heding and Panning (1954), with long, paired posterior prolongations. The same shape was observed in the species we describe here as new. This shape does not agree with most of the other present nominal species of Lipotrapeza. However, in L. littoralis sp. nov., although most specimens have the same calcareous ring shape as L. japonica, one specimen (WMNHINV-2014-101, paratype) had a short calcareous ring with short posterior prolongations (Fig. 6A), and this specimen was apparently in a recovery process caused by evisceration. From this observation, we suggest that the difference in long $v s$. short posterior prolongations is not a fundamental difference in the structure of the calcareous ring, and we consider that the new species must be included in Lipotrapeza because it agrees with the rest of the diagnosis of Clark (1938), as follows: 20 tentacles; body wall lacking ossicles in the middle part; rosettes and rods in the introvert and other parts; pedicels scattered without definite arrangement; tables in the anal tip.

Among these features, the most important common characteristic may be "body wall lacking ossicles in middle part." In fact, if this feature and the shape of the calcareous ring were excluded from consideration, it would be difficult to draw a definite line of demarcation between Lipotrapeza and other phyllophorid genera having 20 tentacles. Under the family Phyllophoridae, Phyllophorella Heding and Panning, 1954 and Phyrella Heding and Panning, 1954 have body wall ossicles that resemble those observed in the early growth stages of Lipotrapeza as described by O'Loughlin et al. (2012). Recent DNA analysis (Michonneau and Pauly 2014), however, has revealed that Phyllophorella and Phyrella are different from Lipotrapeza, and Phyrella has a calcareous ring with long inter-radial elements consisting of many pieces, an arrangement that does not resemble that of Lipotrapeza (Michonneau and Pauly 2014).

From a survey of the literature, one species of the genus Phyllophorella, the Chinese species Phyllophorella liuwutiensis (Yang, 1937) (q.v.), has very similar properties to Lipotrapeza littoralis sp. nov. Phyllophorella liuwutiensis has a calcareous ring that is very similar to that of L. littoralis, and the external morphology and ossicle morphology are also very similar to that of L. littoralis sp. nov. However, both species are distinguishable from each other because Phyllophorella liuwutiensis has tables in the body integument, and the examined specimen of Phyllophorella liuwutiensis is over $200 \mathrm{~mm}$, larger than the present specimens of L. littoralis sp. nov. Furthermore, because Phyllophorella liuwutiensis does not have supporting plates in the pedicels and only has supporting rods, it will also be distinguishable from the present new species on that basis.

We also compared the present new species with the previous nominal species of Lipotrapeza (Paulay 2015): L. capilla Cherbonnier, 1958 (q.v.); L. eichleri O'Loughlin, Barmos and VandenSpiegel, 2012 (q.v.); L. incurva Cherbonnier, 1988 (q.v.); L. litusi O'Loughlin, Barmos and VandenSpiegel, 2012; and L. vestiens (Joshua, 1914) (q.v.). All five species listed above are known from the southern 
A

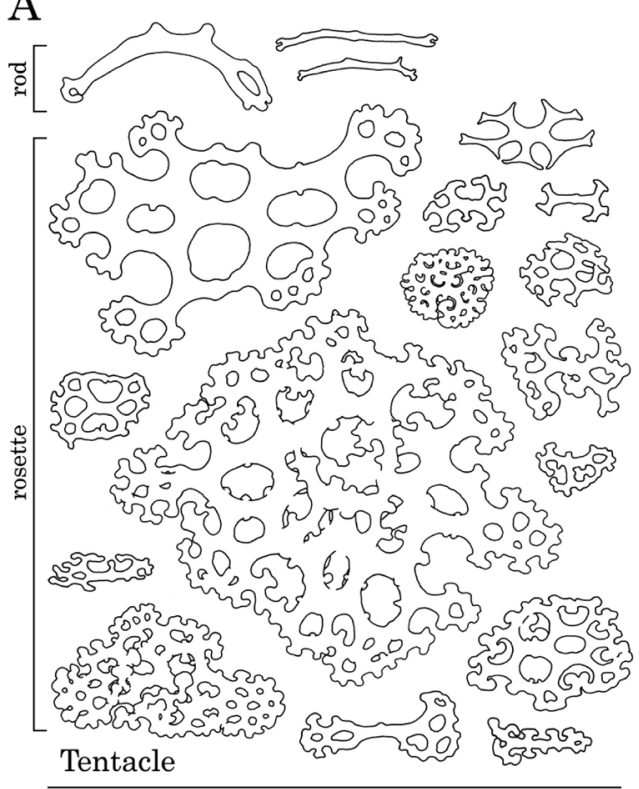

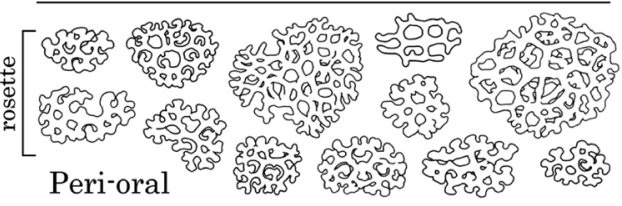

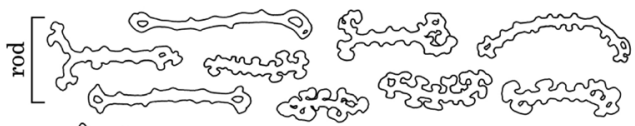

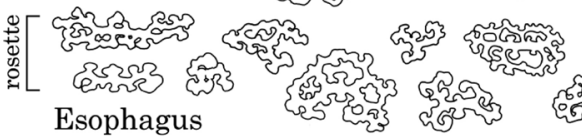

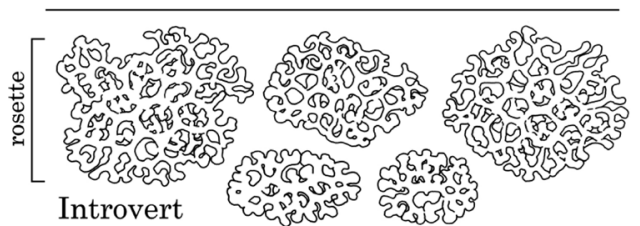

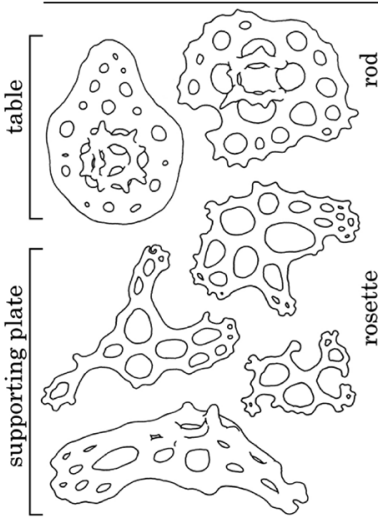

Anal papilla and skin

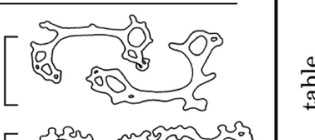

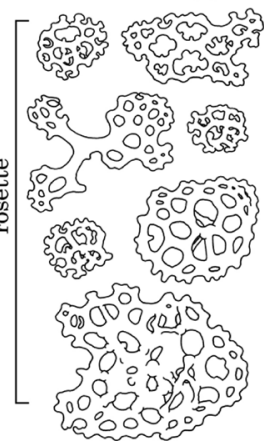

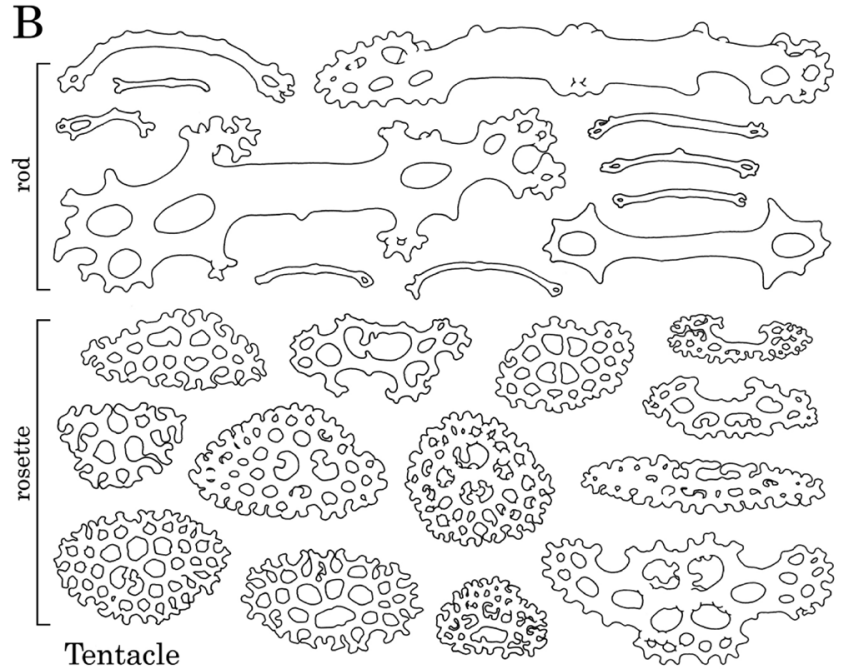

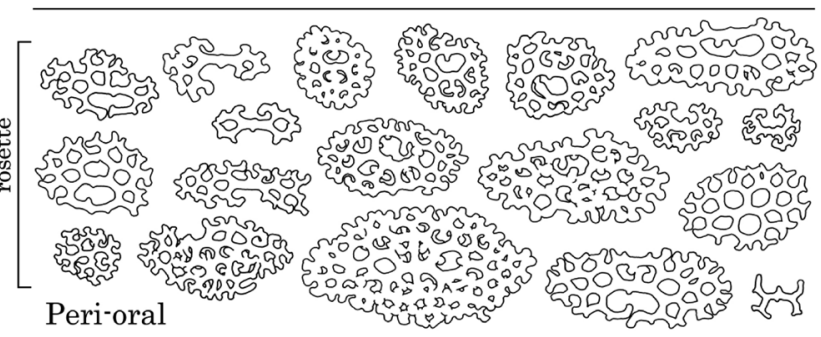

[य

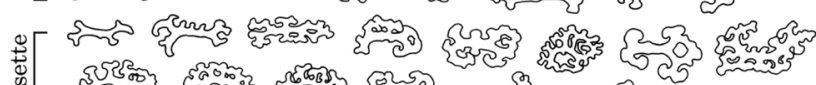

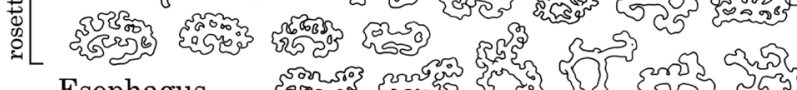

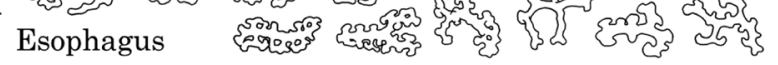
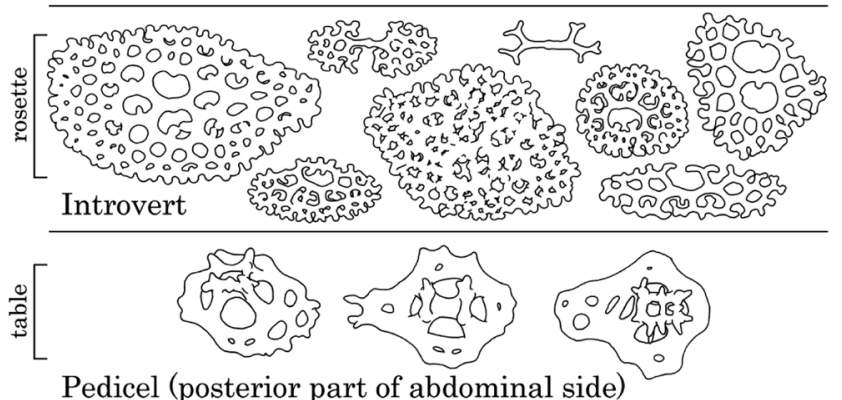

Pedicel (posterior part of abdominal side)

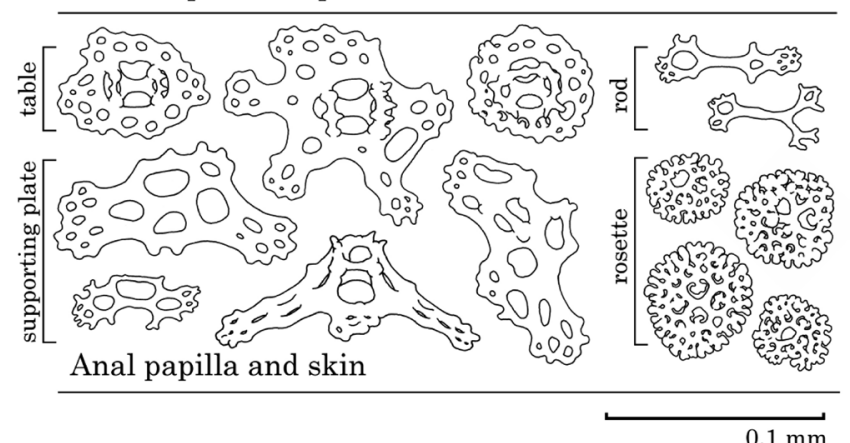

Fig. 7. Variations in the ossicles, Lipotrapeza littoralis sp. nov. Ossicles of the mid-parts of the inter-radial tentacle on the abdominallateral side; the peri-oral skin on the abdominal-lateral side; the skin of the esophagus on the abdominal-lateral side; the middle part of the skin of the introvert in the inter-radius on the abdominal-lateral side; the pedicel of the abdominal side in the radius; appendage and skin around the anus on the abdominal-lateral side. A, WMNH-INV-2014-67 (holotype); B, WMNH-INV-2014-69 (paratype). 


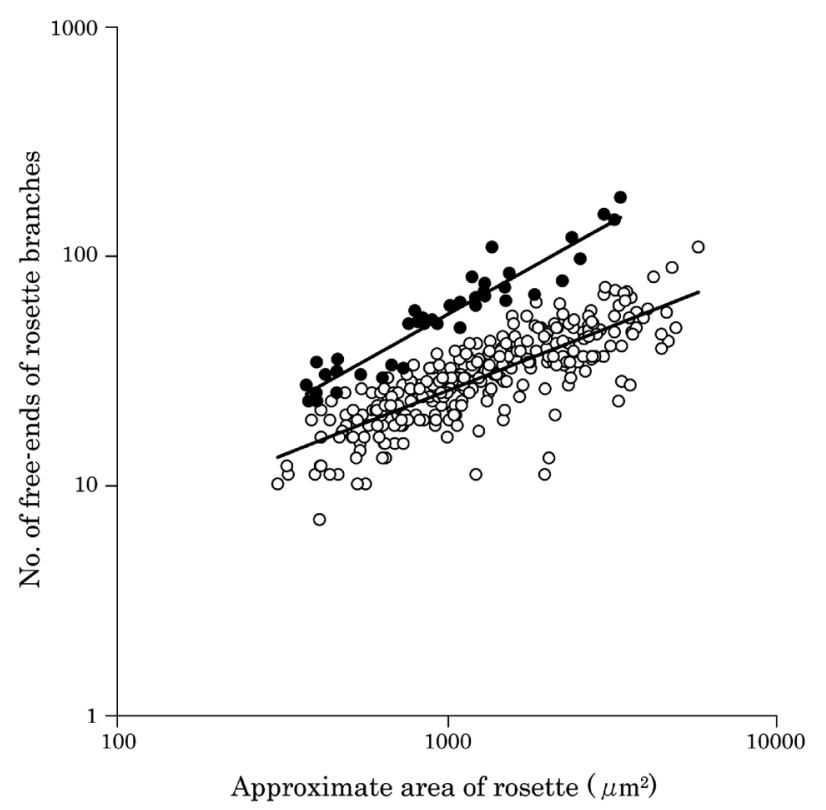

Fig. 8. Relationship between the approximate area of the rosettes and the number of free-ends of rosette branches in Lipotrapeza littoralis sp. nov. (white dots), and in L. japonica Heding and Panning, 1954 (black dots). The solid lines are the regression lines represented by equations (1) and (2) in the text.

hemisphere, and are distant from Japanese waters. Among these, L. capilla and L. incurva were previously judged as not fitting the diagnosis of Clark (1938) (O'Loughlin, Barmos and VandenSpiegel 2012); however, we treat them temporarily as members of Lipotrapeza.

From our survey of the literature of these five species, we found the following: 1) L. capilla has supporting plates in pedicels; a long calcareous ring with long posterior prolongations; one Polian vesicle and one stone canal; and rosette ossicles with many branches in the inner area and a distorted stem (Cherbonnier 1958). This species does not, however, have anything similar to tables anywhere in the body (Cherbonnier 1958). 2) L. eichleri has tables in its young stage; rosette ossicles with overlapping branches in the inner area; and 1-3 Polian vesicles (O'Loughlin, Barmos and VandenSpiegel 2012). It does not, however, have supporting plates in the pedicels, nor external anal scales (O'Loughlin, Barmos and VandenSpiegel 2012). 3) L. incurva has rosette ossicles with few branches in the inner area and a linear stem, and one Polian vesicle (Cherbonnier 1988). It does not, however, have anything related to tables anywhere in the body, nor does it have supporting plates in the pedicels (Cherbonnier 1988). 4) L. litusi has rosette ossicles with overlapped branching in the inner area; one Polian vesicle; and one stone canal (O'Loughlin, Barmos and VandenSpiegel 2012). It does not, however, have anything related to tables anywhere in the body; its pedicels rarely have supporting plates, but mostly have supporting rods and many rosettes; furthermore, its pedicels additionally present in introvert (O'Loughlin, Barmos and VandenSpie- gel 2012). 5) L. vestiens has tables in its young stage, and 1-5 Polian vesicles (O'Loughlin, Barmos and VandenSpiegel 2012). It does not, however, have supporting plates in the pedicels, nor external anal scales (O'Loughlin, Barmos and VandenSpiegel 2012). Consequently, all five of these species are different from L. japonica and L. littoralis sp. nov.

The external morphology of Lipotrapeza japonica and L. littoralis sp. nov. differs in the number of anal appendages, and there is also a possibility that the body colors are different and may be useful delimiting features. Furthermore, the size range of the ossicles from the anal papilla was larger in L. japonica than in L. littoralis sp. nov., although we could not apply statistical analysis because only one individual of L. japonica was examined. We expect that the size ranges of some ossicles will be useful delimiting features. For example, the size ranges of the ossicles from the esophagus and anal periphery of L. littoralis sp. nov. may not be affected much by individual growth, whereas the ossicles from pedicels may be easily affected (Table 2-4). Furthermore, the appearance of the rosette ossicles differs between the two species: the central stems and the densities of the free-ends of branches are different in many cases.

In the original diagnosis of Clark (1938), the shape of the calcareous ring is the most important diagnostic character for the genus Lipotrapeza. It is not entirely clear, however, how "the shape of calcareous ring" should be defined. Because of this problem of ambiguous judgment, Heding and Panning (1954) assigned L. japonica to this genus, probably because they regarded "body wall lacking ossicles in middle part" as of major importance. Following them, we assigned the present new species to this genus because we consider it a close relative of $L$. japonica. To give a strict definition of this genus, it is necessary to accumulate detailed information on the other members of Lipotrapeza, and future studies using DNA analysis are also needed.

\section{Acknowledgements}

We would like to thank the entire staff of the Saikai Pearl Sea Resort, Umi Kirara Aquarium, for their invaluable assistance during this study. We are grateful to Dr. Tom Schiøtte for allowing us to examine the syntypes of $L$. japonica deposited in ZMUC, Mr. Koichi Miki for providing information on sea-cucumber habitats, and $\mathrm{Mr}$. Tohru Imaoka for his helpful advice. We also thank two anonymous reviewers for reviewing the manuscript. This work was partially supported by the Research Institute of Marine Invertebrates (Tokyo) under grant 2015KO-1. Financial support for part of the microscopic observations was provided by Mr. Shinichi Ikebe of the Supporters' Association of WMNH. 


\section{References}

Cherbonnier G (1958) Holothuries des côtes de Sierra-Leone, $4^{\mathrm{e}}$ note. Bulletin du Muséum national d'Historie naturella, Paris 30: 294-299.

Cherbonnier G (1988) Echinoderms: Holothurides. Faune de Madagascar 70: 1-292.

Clark HL (1938) Echinoderms from Australia. Memoirs of the Museum of Comparative Zoology at Harvard College 55: $1-596$.

Heding SG, Panning A (1954) Phyllophoridae. Spolia Zoologica Musei Hauniensis 13: 1-209.

Imaoka T (1995) Holothuroidea. In: Genshoku Kensaku Nihon Kaigan Doubutsu Zukan. Vol. II. [Guide to Seashore Animals of Japan with Color Pictures and Keys. Vol. II.] (ed Nishimura S). Hoikusha, Osaka, pp. 553-572. (In Japanese)

Joshua EC (1914) Victorian Holothuroidea, with descriptions of new species. Proceedings of the Royal Society of Victoria 27: $1-11$.

Michonneau F, Pauly G (2014) Revision of the genus Phyrella (Holothuroidea: Dendrochirotida) with the description of a new species from Guam. Zootaxa 3760: 101-140.

Ohshima H (1912) On the system of Phyllophorinae with descriptions of the species found in Japan. Annotationes zoologicae Japonenses 8: 53-96.

O’Loughlin PM, Barmos S, VandenSpiegel D (2012) The phyllophorid sea cucumbers of southern Australia (Echinodermata: Holothuroidea: Dendorochirotida: Phyllophoridae). Memoirs of Museum Victoria 69: 269-308.

Paulay G (2015) Lipotrapeza Clark, 1938. Accessed through: World Register of Marine Species at http://www.marinespecies. org/aphia.php?p=taxdetails\&id=528791 (16 July 2016).

Yang PF (1937) Report on the holothurians from the Fukien Coast. Bulletin Marine Biology of Amoy China 2: 1-46. 